\title{
Multi-black Holes and Earthquakes on Riemann Surfaces with Boundaries
}

\author{
Francesco Bonsante $^{1}$, Kirill Krasnov ${ }^{2}$, and Jean-Marc Schlenker ${ }^{3}$ \\ ${ }^{1}$ Dipartimento di Matematica dell'Università degli Studi di Pavia, via \\ Ferrata 1, 27100 Pavia, Italy, ${ }^{2}$ School of Mathematical Sciences, \\ University of Nottingham, Nottingham, NG7 2RD, UK, and ${ }^{3}$ Institut de \\ Mathématiques de Toulouse (UMR CNRS 5219), Université Paul Sabatier, \\ 31062 Toulouse cedex 9, France
}

Correspondence to be sent to: jmschlenker@gmail.com

We prove an "Earthquake Theorem" for hyperbolic metrics with geodesic boundary on a compact surface $S$ with boundary: given two hyperbolic metrics with geodesic boundary on a surface with $k$ boundary components, there are $2^{k}$ right earthquakes transforming the first in the second. An alternative formulation arises by introducing the enhanced Teichmüller space of $S$ : we prove that any two points of the latter are related by a unique right earthquake. The proof rests on the geometry of "multi-black holes," which are three-dimensional Anti-de Sitter manifolds, topologically the product of a surface with boundary by an interval.

\section{Introduction}

The Earthquake Theorem. Let $\Sigma$ be a closed surface, with a hyperbolic metric $g$, let $c$ be a simple closed geodesic on $(\Sigma, g)$, and let $l$ be a positive real number. The image of $g$ by the right earthquake of length $l$ along $c$ is the hyperbolic metric obtained by cutting $\Sigma$ along $c$ and gluing back after rotating the "left" side of $c$ by $l$. This defines a map from the Teichmüller space $\mathcal{T}_{\Sigma}$ of $\Sigma$ to itself. 
Suppose now that $\lambda$ is a measured geodesic lamination on $(\Sigma, h)$ which is ratio$n a l$, that is, its support is a disjoint union of closed curves $c_{1}, \cdots, c_{n}$. The transverse measure is then described by a set of positive numbers $l_{1}, \cdots, l_{n}$ associated to the $c_{i}$. The image of $g$ by the right earthquake along $\lambda$ is obtained as above, by doing a "fractional Dehn twist" along each of the $c_{i}$, with a length parameter given by the $l_{i}$. Again this defines a map from $\mathcal{T}_{\Sigma}$ to itself.

Thurston $[19,20]$ discovered that this definition can be extended by continuity to all measured geodesic laminations on $(\Sigma, g)$. In other terms, it makes sense to talk about the right earthquake along any measured geodesic lamination on $(\Sigma, g)$. This defines a map:

$$
E_{\mathrm{r}}: \mathcal{M} \mathcal{L}_{\Sigma} \times \mathcal{T}_{\Sigma} \rightarrow \mathcal{T}_{\Sigma}
$$

where $\mathcal{M} \mathcal{L}_{\Sigma}$ is the space of measured laminations on $\Sigma$. Thurston also discovered a striking feature of this earthquake map.

Theorem 1.1 (Thurston $[20,16])$. For any $h, h^{\prime} \in \mathcal{T}_{\Sigma}$, there exists a unique $\lambda \in \mathcal{M} \mathcal{L}_{\Sigma}$ such that $E_{\mathrm{r}}(\lambda)(h)=h^{\prime}$.

Earthquakes on surfaces with boundary. Let now $\Sigma$ be a compact orientable surface of genus $g$ with $n$ boundary components. We will assume $\Sigma$ to have negative Euler characteristic

$$
\chi(\Sigma)=2-2 g-n<0
$$

Let $\mathcal{T}_{g, n}$ be the Teichmüller space of hyperbolic metrics on $\Sigma$ with geodesic boundary (such that each geodesic boundary component is a closed curve), considered up to isotopy. $\mathcal{T}_{g, n}$ is a contractible manifold of dimension $6 g-6+3 n$.

We also consider the space $\mathcal{M} \mathcal{L}_{g, n}$ of measured laminations on the interior of $\Sigma$, see for example [12] (a precise definition is given in Section 3). Note that the transverse weight on those laminations is required to be finite on any closed transverse segment in the interior of $\Sigma$, but the weight might be infinite on segments with an endpoint on the boundary of $\Sigma$, see Figure 1 . Given a measured lamination $\lambda \in \mathcal{M} \mathcal{L}_{g, n}$ and a hyperbolic metric $h \in \mathcal{T}_{g, n}$, there is a unique way to realize $\lambda$ as a measured geodesic lamination on $(\Sigma, h)$.

The main result presented here is the following. 


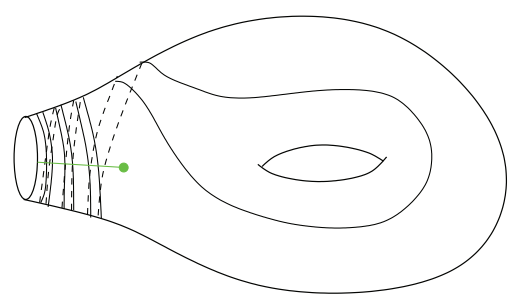

Fig. 1. An example of a geodesic lamination on a surface with a geodesic boundary. The geodesics forming the lamination can spiral on to the boundary. The total weight of an arc ending at the boundary (as shown in green here) is allowed to be infinite.

Theorem 1.2. Given $h_{1}, h_{2} \in \mathcal{T}_{g, n}$, there are exactly $2^{n}$ measured laminations $\lambda_{1}, \cdots, \lambda_{2^{n}}$ on the interior of $\Sigma$ such that the right earthquake along the $\lambda_{i}$ sends $h_{1}$ to $h_{2}$.

This result extends to the hyperbolic metrics with some geodesic boundary components and some cusps, however the number of possible measured laminations is lower when one of the boundary components corresponds to a cusp for either $h_{1}$ or $h_{2}$. The statement of Theorem 1.2 looks simple, but it might be less obvious than it first seems; even the case $g=0, n=3$ (for a hyperbolic pair of pants), where everything can be described explicitly, displays some interesting phenomena. This case is described in details at the end of Section 2 (see Proposition 2.4 and the paragraph right before Section 3). The enhanced Teichmüller space. The fact that the number of right earthquakes sending a given hyperbolic metric to another one is $2^{n}$ rather than one can appear distressing at first sight. There is a simple geometric formalism, however, under which this disagreement disappears. It is based on a definition due to V. Fock $[13,14,15,7]$ which appeared naturally in different contexts. The terminology is borrowed from Bonahon and Liu [7].

Definition 1.3. The enhanced Teichmüller space of $\Sigma, \hat{\mathcal{T}}_{g, n}$, is the space of $n+1$-uples $\left(h, \epsilon_{1}, \cdots, \epsilon_{n}\right)$, where $h$ is a hyperbolic metric with geodesic boundary on $\Sigma$ and, for each $k \in\{1, \cdots, n\}, \epsilon_{k}$ is:

- 0 if the corresponding boundary component of $\Sigma$ corresponds to a cusp of $h$,

- either + or - if the corresponding boundary component of $\Sigma$ corresponds to a geodesic boundary component of $h$.

Fock showed in particular that shear coordinates on a surface with some boundary components provide a natural parameterization of this enhanced Teichmüller space. 


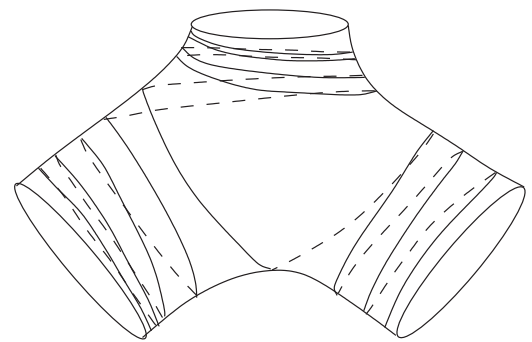

(a)

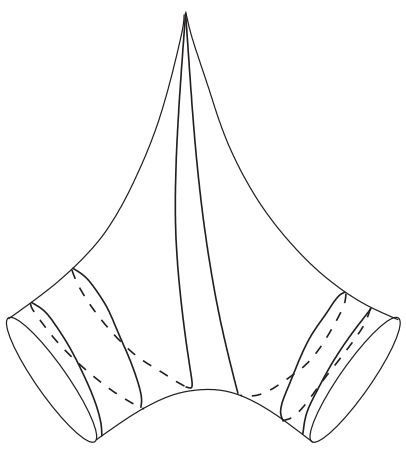

(b)

Fig. 2. A boundary component can degenerate into a puncture as a result of an earthquake. Figure (a) shows the surface before and (b) after an earthquake.

Note that the boundary of $\mathcal{T}_{g, n}$ has a stratified structure, with strata corresponding to subsets of the set of boundary components which are "pinched" to obtain cusps, as shown in Figure 2. Heuristically, $\hat{\mathcal{T}}_{g, n}$ is obtained by "reflecting" $\mathcal{T}_{g, n}$ along the codimension 1 strata of its boundary, and $\hat{\mathcal{T}}_{g, n}$ contains an open dense subset which is a $2^{n}$-fold cover of the interior of $\mathcal{T}_{g, n}$. There is also a natural embedding of $\mathcal{T}_{g, n}$ in $\hat{\mathcal{T}}_{g, n}$, obtained by taking all $\epsilon_{i}$ equal to + in the definition above.

It is possible to define in a rather natural-but perhaps not obvious-way the element of $\hat{\mathcal{T}}_{g, n}$ obtained by an earthquake along a measured geodesic lamination, that is, a map $E_{\mathrm{r}}: \mathcal{M} \mathcal{L}_{g, n} \times \hat{\mathcal{T}}_{g, n} \rightarrow \hat{\mathcal{T}}_{g, n}$. This map has the key properties that should be required of it:

- its restriction to $\mathcal{T}_{g, n}$ (considered as a subset of $\mathcal{T}_{g, n}$ ), followed by the projection from $\hat{\mathcal{T}}_{g, n}$ to $\mathcal{T}_{g, n}$, is the right earthquake map $E_{\mathrm{r}}: \mathcal{M} \mathcal{L}_{g, n} \times \mathcal{T}_{g, n} \rightarrow \mathcal{T}_{g, n}$ defined above,

- it is continuous,

- for any $\lambda \in \mathcal{M} \mathcal{L}_{g, n}$, any $h \in \hat{\mathcal{T}}_{g, n}$, and any $t, t^{\prime} \in \mathbb{R}_{>0}$,

$$
\left(E_{\mathrm{r}}(t \lambda) \circ E_{\mathrm{r}}\left(t^{\prime} \lambda\right)\right)(h)=E_{\mathrm{r}}\left(\left(t+t^{\prime}\right) \lambda\right)(h)
$$

Theorem 1.2 can then be reformulated in a simpler way in terms of $\hat{\mathcal{T}}_{g, n}$.

Theorem 1.4. For any $h, h^{\prime} \in \hat{\mathcal{T}}_{g, n}$, there exists a unique $\lambda \in \mathcal{M} \mathcal{L}_{g, n}$ such that $h^{\prime}=$ $E_{\mathrm{r}}(\lambda)(h)$. 
It is shown in Section 9 how Theorem 1.4 follows from Theorem 1.2. Note that some care is needed there to give the proper definitions and prove the result.

The Mess proof of the Earthquake Theorem. G. Mess [17] discovered some striking similarities between quasifuchsian hyperbolic 3-manifolds and the so-called GHMC (for "globally hyperbolic compact maximal") AdS (for "Anti-de Sitter") three-dimensional manifolds. As a consequence, he found a direct and very geometric proof of the Earthquake Theorem.

The three-dimensional AdS space, $\mathrm{AdS}_{3}$, can be defined as a quadric in $\mathbb{R}^{4}$ endowed with a symmetric bilinear form of signature $(2,2)$, with the induced metric:

$$
\operatorname{AdS}_{3}=\left\{x \in \mathbb{R}^{2,2} \mid\langle x, x\rangle=-1\right\} .
$$

It is a complete Lorentz space of constant curvature -1 , analog in certain ways to the hyperbolic 3-space. Defined in this way, $\mathrm{AdS}_{3}$ is however not simply connected, its fundamental group is $\mathbb{Z}$. Its totally geodesic planes are isometric to $H^{2}$, while its time-like geodesics are closed of length $2 \pi$.

An AdS manifold is a manifold endowed with a Lorentz metric locally isometric to the metric on $\mathrm{AdS}_{3}$. Recall that a Cauchy surface in a Lorentz manifold is a surface which intersects each inextendible time-like geodesic exactly once, see for example [18]. We are particularly interested here in globally hyperbolic maximal compact (GHMC) AdS 3-manifolds: those AdS 3-manifolds which contain a closed, space-like Cauchy surface, and which are maximal under these conditions (any isometric embedding into an AdS manifold containing a closed Cauchy surface is an isometry). GHMC AdS manifolds display some striking similarities with quasifuchsian hyperbolic 3-manifolds.

Mess discovered in particular that the space of GHMC AdS manifolds which are topologically $\Sigma \times \mathbb{R}$ (where $\Sigma$ is a closed surface of genus at least 2) is parameterized by the product of two copies of the Teichmüller space of $\Sigma, \mathcal{T}_{\Sigma}$. This is strongly reminiscent of the Bers double uniformization theorem [6]. However, it does not involve a conformal structure at infinity, but rather the "left" and "right" hyperbolic metrics, $h_{1}$ and $h_{\mathrm{r}}$, associated to such an AdS 3-manifold (the definitions can be found in Section 2).

Moreover, those GHMC AdS manifolds have a "convex core," and the boundary of this convex core has two connected components, each with an induced hyperbolic metric (which we call $\mu_{+}$and $\mu_{-}$) and a measured bending lamination (called $\lambda_{+}$and $\lambda_{-}$here). The left hyperbolic metric $h_{1}$ is obtained from the induced metric on the upper boundary component of the convex core, $\mu_{+}$, by the action of the left earthquake relative to $\lambda_{+}$ (rather than by a grafting along $\lambda_{+}$, as in the quasifuchsian context). This leads to the 
following diagram, where $E_{1}(\lambda)$ (resp. $E_{\mathrm{r}}(\lambda)$ ) is the left (resp. right) earthquake relative to the measured lamination $\lambda$.

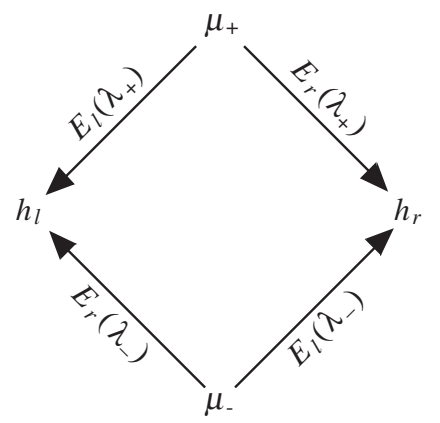

It follows that $h_{\mathrm{l}}=E_{\mathrm{l}}\left(2 \lambda_{+}\right)\left(h_{\mathrm{r}}\right)=E_{\mathrm{r}}\left(2 \lambda_{-}\right)\left(h_{\mathrm{r}}\right)$. Since any couple $\left(h_{\mathrm{l}}, h_{\mathrm{r}}\right)$ can be obtained as the left and right hyperbolic metrics of exactly one GHMC AdS manifold, a simple proof of the Earthquake Theorem follows.

This line of ideas can be extended to obtain an "Earthquake Theorem" for hyperbolic metrics with cone singularities, of fixed angle in $(0, \pi)$, on closed surfaces, see [9]. The GHMC AdS manifolds considered by Mess are then replaced by similar manifolds with "particles," that is, cone singularities along time-like geodesic segments.

Multi-black holes. There is a class of three-dimensional AdS manifolds analogous to GHMC manifolds, which is obtained by replacing the closed Cauchy surface by a noncompact one. These manifolds were first defined in the physics literature $[1,10]$ and are called "multi-black holes" (called MBH here). A mathematical description can be found in $[3,4]$. The simplest example is obtained from a complete hyperbolic metric $h$ on a compact surface $S$ of genus $g$ with $n$ disks removed (with each end of infinite area) by a warped product construction:

$$
M=\left(S \times(-\pi / 2, \pi / 2),-d t^{2}+\cos (t)^{2} h\right) .
$$

More general MBH metrics are obtained by deforming those examples, losing the symmetry $t \mapsto-t$.

It is in particular proved in [3, 4] that, given a compact surface with boundary $S$, the space of MBHs which are topologically the product of $S$ by an interval is parameterized by the product of two copies of the Teichmüller space of hyperbolic metrics with geodesic boundary on $S$, as was proved by Mess for closed surfaces [17].

The geometry of multi-black holes and the idea of the proof. Let $M$ be an $\mathrm{MBH}$, with fundamental group $\pi_{1}(\Sigma)$. The main idea of the proof of Theorem 1.2 is to consider a 
special class of convex pleated surfaces in a $\mathrm{MBH}$. It was proved in [5] that given a $\mathrm{MBH} M$ with right and left holonomies $h_{\mathrm{l}}$ and $h_{\mathrm{r}}$, there is a one-to-one correspondence between

- space-like, convex, pleated, inextendible surfaces in $M$ (in general not complete, but with geodesic boundary),

- earthquakes between pairs of hyperbolic surfaces with convex boundary (of finite or infinite area, possibly with vertices at infinity) with left and right holonomies equal to $h_{1}$ and $h_{\mathrm{r}}$.

One key technical result here is that, given $M$, there is a finite number of convex pleated surfaces for which each boundary component is either a closed geodesic or a cusp. Those surfaces have a simple characterization in terms of the quotient of the boundary components of the convex hull of some natural curves complementing the limit set of $M$ in a "boundary at infinity" of $\mathrm{AdS}_{3}$ (see the first paragraph of Section 3), as shown in Proposition 8.1.

In a previous version of this paper, multi-black holes played a key role in the proof of the main result. Here however this proof has been rewritten to be readable to readers with no previous knowledge of multi-black holes. Some elements of the geometry of multi-black holes, and the relation with the main theorem here, are explained in Section 10.

A description in terms of measured laminations. A by-product of the arguments used for the proof of Theorem 1.2 is another description of the space of MBHs of given topology, based on pleated surfaces or, in other terms, on hyperbolic metrics and measured laminations on compact surfaces with boundary. This is explained in more details in the physics introduction of a previous version of this text, see [8]. We do not dwell on this point here.

\section{Earthquakes on $\mathcal{T}_{g, n}$}

\subsection{The Teichmüller space $\mathcal{T}_{g, n}$}

A hyperbolic metric $\eta$ on $\Sigma$ is said to be admissible if:

(1) It has a finite area.

(2) Its completion has a geodesic boundary.

(3) Each geodesic boundary component is a closed curve. 
We denote by $\Sigma_{\eta}$ the hyperbolic surface $(\Sigma, \eta)$, and by $\bar{\Sigma}_{\eta}$ the completion of $\Sigma_{\eta}$. Notice that the topological type of $\bar{\Sigma}_{\eta}$ depends on $\eta$. A neighborhood of a puncture can look like either a cusp or a neighborhood of a boundary component.

The Teichmüller space $\mathcal{T}_{g, n}$ for $\Sigma$ is the space of admissible hyperbolic metrics up to the action of diffeomorphisms isotopic to the identity. For $\chi(\Sigma)<0$, this space is non-empty.

Given an admissible metric on $\Sigma$, its holonomy is a faithful (i.e., injective) and discrete representation

$$
h: \pi_{1}(\Sigma) \rightarrow P S L_{2}(\mathbb{R}) .
$$

The surface $\bar{\Sigma}$ is the convex core $\mathcal{K}$ of the quotient of $\mathbb{H}^{2}$ (hyperbolic plane) by the action of $\Gamma:=h\left(\pi_{1}(\Sigma)\right)$. One can easily check that the following statement holds

For each $\gamma \in \pi_{1}(\Sigma)$ parallel to a puncture, either $h(\gamma)$ is parabolic or its axis is a boundary curve of $\left.\mathcal{K} .{ }^{*}\right)$

A faithful and discrete representation $h: \pi_{1}(\Sigma) \rightarrow P S L_{2}(\mathbb{R})$ satisfying $\left(^{*}\right)$ is called admissible. Thus, the holonomy of an admissible metric is an admissible representation. Conversely, the quotient of the convex core of an admissible representation is a finite area hyperbolic surface homeomorphic to $\Sigma$. Thus, the space $\mathcal{T}_{g, n}$ can be identified with the space of admissible representations of $\pi_{1}(S)$ into $P S L_{2}(\mathbb{R})$, up to conjugacy. Since the fundamental group of $\Sigma$ is a free group on $2 g+n-1$ generators, it follows that the space of representations of $\pi_{1}(\Sigma)$ into $P S L_{2}(\mathbb{R})$ is $P S L_{2}(\mathbb{R})^{2 g+n-1}$. Taking into account the fact that conjugate representations lead to the same metrics, we see that $\operatorname{dim} \mathcal{T}_{g, n}=6 g-6+3 n$. The Teichmüller space $\mathcal{T}_{g, n}$ is a closed subset of this space with the interior corresponding exactly to the metrics without cusps. The boundary of $\mathcal{T}_{g, n}$ corresponds to structures with some cusps.

\subsection{Measured geodesic laminations on a hyperbolic surface with geodesic boundary}

Let us fix an admissible metric $\eta \in \mathcal{T}_{g, n}$ with holonomy $h: \pi_{1}(\Sigma) \rightarrow P S L_{2}(\mathbb{R})$.

A geodesic lamination on $\Sigma_{\eta}$ is a closed subset $L$ foliated by complete geodesics. A leaf of $L$ is a geodesic of the foliation, whereas a stratum is either a leaf or a connected component of $\Sigma_{\eta} \backslash L$. 
Since the area of $\Sigma_{\eta}$ is finite, the structure of $L$ can be proved to be similar to the structure of a geodesic lamination on a closed surface. In particular:

- The Lebesgue measure of $L$ is 0 .

- There exists a unique partition of $L$ in complete geodesics (i.e., the support $L$ is sufficient to encode the lamination).

- $\Sigma_{\eta} \backslash L$ contains finitely many connected components. Each of them is isometric to (the interior of) a finite area hyperbolic surface with geodesic boundary.

A leaf of $L$ is a boundary curve if it is the boundary of some component of $\Sigma_{\eta} \backslash L$.

- Boundary curves are finitely many. Moreover, they are dense in $L$.

The following lemma describes the behavior of a geodesic lamination near a puncture.

Lemma 2.1. For each boundary component $c$ there exists an $\varepsilon$-neighborhood $U$ such that every leaf intersecting $U$ must spiral around $U$. Moreover, leaves in $U \cap L$ are locally isolated.

The same result holds for cusps, by exchanging $\varepsilon$-neighborhoods by horoballs: for each cusp $c^{\prime}$ there exists a neighborhood $U$ bounded by a horocycle $C$ such that every leaf intersecting $C$ does so orthogonally, and leaves in $U \cap C$ are locally isolated.

Proof. We prove the first part of the statement. The case with cusp is completely analogous. On the other hand, the proof uses the same arguments used in [11] to describe the behavior of a geodesic lamination (without measure) on a closed surface in a regular neighborhood of some closed leaf.

Let $\Sigma_{\eta}=\mathcal{H} / h$ where $h$ is the holonomy representation of $\pi_{1}(\Sigma)$ and $\mathcal{H}$ is the convex core of $h$.

Let $\tilde{L}$ be the pre-image of $L$ on $\mathbb{H}^{2}, \tilde{c}$ be a pre-image of $c$, and $\gamma$ be a generator of the stabilizer of $c$. If $d$ is the length of $c$, we may find $\varepsilon>0$ such that if $\tilde{c}$ is a complete geodesic disjoint from $\tilde{C}$ and $\varepsilon$-close to $\tilde{C}$ then the length of the projection of $\tilde{C}$ on $\tilde{c}$ is greater than $d$. Thus, if $\tilde{c}^{\prime}$ is at positive distance from $\tilde{c}$ then $\gamma \tilde{c}^{\prime}$ must intersect $\tilde{c}$.

Thus, leaves of $L$ intersecting $U_{\varepsilon}$ have to spiral around $c$.

Now let us prove that leaves in $U \cap L$ are locally isolated. By taking a smaller $\varepsilon$, we may suppose that $U_{\varepsilon}$ projects on a regular neighborhood of $c$. Take a leaf spiraling around $c$, say $l$, and denote by $\tilde{l}$ a lifting of $l$ on $\mathbb{H}^{2}$ intersecting $U_{\varepsilon}$. Suppose that between $l$ and $\gamma l$ there are infinitely many leaves intersecting $U_{\varepsilon}$. Thus, there are infinitely many 
boundary leaves. On the other hand, leaves between $l$ and $\gamma l$ intersecting $U_{\varepsilon}$ are not permuted by $\pi_{1}(\Sigma)$, so we get a contradiction. (It follows from this argument that there are finitely many boundary leaves in $\Sigma$.)

Transverse measures. The notion of transverse measure can be introduced as in the closed case. We say that an $\operatorname{arc}$ in $c$ is transverse to $L$ if it is transverse to the leaves of $L$.

A transverse measure on $L$ is the assignment of a Borel measure $\mu_{c}$ on each transverse $\operatorname{arc} c$ such that:

(1) The support of $\mu_{C}$ is $c \cap L$.

(2) If $c^{\prime} \subset c$, then $\mu_{c^{\prime}}=\left.\mu_{c}\right|_{c^{\prime}}$.

(3) If two transverse arcs are homotopic through a family of transverse arcs, then their total masses are equal.

The simplest example of a geodesic lamination is a simple geodesic $u$. In such a case, a measure $\mu_{c}$ is concentrated on the intersection points of $c$ with $u$. The mass of each single intersection point is a number independent of $c$ and is, by definition, the weight of $u$. Thus, transverse measures on $u$ are encoded by a positive number.

On closed surfaces, every measured geodesic lamination splits as the disjoint union of sub-laminations

$$
L=S \cup L_{1} \cup L_{2} \cup \ldots \cup L_{k}
$$

such that the support of $S$ is a finite union of simple geodesics and each leaf $l \subset L_{i}$ is dense in $L_{i}$.

In the case we are concerned with, things are a bit more complicated, since $L$ is not supposed to be compact. On the other hand, we have seen that near a puncture $L$ has a simple behavior. Notice that a consequence of Lemma 2.1 is that every geodesic in $L$ that enters a cusp or spirals around a geodesic boundary is weighted. Thus, it cannot have accumulation points in $\Sigma$. It follows that such leaves are properly embedded in $\Sigma$. So, if some regular neighborhoods of the punctures are cut off from $\Sigma$, such leaves appear as properly embedded compact arcs.

This remark allows to find a canonical decomposition of a measured geodesic lamination. 
Lemma 2.2. If $\lambda$ is a measured geodesic lamination on $(\Sigma, \mu)$, then it splits as the union of sub-laminations

$$
L=B \cup S \cup L_{1} \cup L_{2} \cup \ldots \cup L_{k}
$$

such that $B$ is the union of leaves that do not have compact closure in $\Sigma, S$ is a union of closed geodesics. $L_{i}$ is compact and every leaf $l$ of $L_{i}$ is dense in $L_{i}$.

Proof. Define first $B$ as the union of the geodesics in the support of $\lambda$ that enter any neighborhood of the boundary. Their behavior near the boundary is described by Lemma 2.1. Let $\lambda^{\prime}$ be the measured lamination obtained by removing from $\lambda$ the measure supported on $B$.

We now consider the surface $\left(\Sigma^{\prime}, \mu^{\prime}\right)$ obtained by gluing two copies of $(\Sigma, \mu)$ along their boundary, by identifying corresponding points of the boundary on the two copies. Since the support of $\lambda^{\prime}$ does not enter some neighborhood of $\partial \Sigma, \lambda^{\prime}$ lifts to a measured geodesic lamination on $\left(\Sigma^{\prime}, \mu^{\prime}\right)$. Applying the known decomposition result for closed surfaces to $\lambda^{\prime}$ on $\Sigma^{\prime}$ shows that its support can be written as $S \cup L_{1} \cup \cdots \cup L_{k}$, and the result for $\lambda$ follows.

Measured geodesic laminations with compact support are well understood. To get a complete description of a general measured geodesic lamination, we should describe complete embedded geodesics of $\Sigma$ that escape from compact sets.

We have seen that every leaf $l$ in $B$ produces a properly embedded arc in the complement of some regular neighborhood of the puncture. Notice that the homotopy class of this arc does not depend on the regular neighborhood. With a slight abuse of language, we say that $l$ represents such a class.

We could expect that $l$ is determined by its homotopy class. This is not completely true. In fact the homotopy class does not "see" in which way $l$ winds around the boundary of $\Sigma_{\eta}$.

Lemma 2.3. Suppose that a positive way of spiraling around each boundary component of $\bar{\Sigma}_{\eta}$ is fixed. Then in each homotopy class of properly embedded arcs joining two punctures of $\Sigma$, there exists a unique geodesic representative that spirals in the positive way. 
Proof. Let $c_{1}$ and $c_{2}$ be two punctures of $\Sigma$, and let $h$ be a homotopy class of properly embedded arc joining them. $c_{1}$ and $c_{2}$ correspond to geodesic boundary components of $\bar{\Sigma}_{\eta}$, which we still call $c_{1}$ and $c_{2}$. Let $c_{1}^{\prime}$ be a lift of $c_{1}$ as a connected component of the (geodesic) boundary of the universal cover of $\bar{\Sigma}_{\eta}$, and similarly let $c_{2}^{\prime}$ be a lift of $c_{2}$ as a connected component of $\partial \bar{\Sigma}_{\eta}$, chosen so that there is a lift $h^{\prime}$ of $h$ as a path connecting $c_{1}^{\prime}$ to $c_{2}^{\prime}$.

Any realization of $h$ as a geodesic spiraling around $c_{1}$ and $c_{2}$ has to lift to the universal cover of $\bar{\Sigma}_{\eta}$ as a geodesic which is asymptotic to $c_{1}^{\prime}$ and $c_{2}$. There are four such geodesics, depending on the choice of one of the two ends of $c_{1}^{\prime}$ and one of the two ends of $c_{2}^{\prime}$. But only one of those choices corresponds to the positive spiraling direction, so there is only one geodesic realization of $h^{\prime}$.

Given an admissible metric $\eta$, denote by $\mathcal{M L}_{g, n}(\eta)$ the set of measured geodesic laminations on the surface $\Sigma_{\eta}$. From Lemmas 2.2 and 2.3, it follows that if $\Sigma_{\eta}$ and $\Sigma_{\eta^{\prime}}$ have no cusp, then there is a natural bijection

$$
\mathcal{M} \mathcal{L}_{g, n}(\eta) \rightarrow \mathcal{M} \mathcal{L}_{g, n}\left(\eta^{\prime}\right)
$$

Actually a measured geodesic lamination $\lambda$ on $\mathcal{M L}_{g, n}(\eta)$ is the union of a compact sublamination $\lambda_{c}$ and a sub-lamination $\lambda_{b}$ of leaves spiraling along some boundary components. Now, there is a compact measured geodesic lamination $\lambda_{c}^{\prime}$ in $\mathcal{M} \mathcal{L}_{g, n}\left(\eta^{\prime}\right)$ obtained by "straightening" leaves of $\lambda_{C}$ with respect to $\eta^{\prime}$ (it is possible for instance to consider $\Sigma$ as included in its double and apply the analogous result for laminations in a closed surface). Moreover by Lemma 2.3, we can also straighten the lamination $\lambda_{b}$ with respect to $\eta$, and the union of $\lambda_{c}^{\prime} \cup \lambda_{b}^{\prime}$ corresponds to $\lambda$ via identification (1).

When $\eta^{\prime}$ is supposed to have some cusps, the map (1) can be defined in the same way, but it is no longer one-to-one. The reason is that if we change the orientation of spiraling of leaves along a geodesic boundary of $\eta$ that is a cusp of $\eta^{\prime}$, the corresponding lamination of $\eta^{\prime}$ does not change at all.

In this work, we will denote by $\mathcal{M L}_{g, n}$ the set of measured geodesic laminations of a hyperbolic surface with geodesic boundary (without cusps). From the above discussion, this set is well defined and for every admissible metric $\eta$ we have a surjective map

$$
\mathcal{M} \mathcal{L}_{g, n} \rightarrow \mathcal{M} \mathcal{L}_{g, n}(\eta)
$$




\subsection{The mass of boundary component}

Given a measured geodesic lamination $\lambda$ on $\Sigma_{\eta}$, the mass of a puncture with respect to $\lambda$ is a positive number $m_{\lambda}(c)$ that measures how much the measured lamination is concentrated in a neighborhood of $c$.

We will give the construction of $m_{\lambda}(c)$, when $c$ corresponds to a geodesic boundary component of $\bar{\Sigma}_{\eta}$.

Fix a regular neighborhood $U_{\varepsilon}$ of $c$ such that every leaf intersecting $U_{\varepsilon}$ spirals around $c$. For every $x \in U_{\varepsilon}$, consider the geodesic loop $c_{X}$ with vertex at $x$ parallel to $c$. We claim that the total mass of such a loop does not depend on $X$.

Let $\mathcal{H}$ be the convex core of the holonomy $h$ of $\Sigma_{\eta}$. Choose a lifting of $c$, say $\tilde{c} \subset \partial \mathcal{H}$ and let $\gamma$ be the generator of the stabilizer of $\tilde{c}$ in $\pi_{1}(\Sigma)$. If $\tilde{X}$ is a lifting of $x$, then the loop $c_{X}$ lifts to the segment $[x, h(\gamma) x]$. Since geodesics spiraling around $c$ lift to geodesics asymptotic to $\tilde{C}$, it follows that $c_{X}$ intersects every such geodesic once. Since the total mass of $c_{X}$ depends only on the number of intersection points of $c_{X}$ with each leaf, it does not depend on $x$.

The same construction works when $c$ corresponds to a cusp.

Notice that $m_{\lambda}(c)=0$ if and only if there exists a neighborhood of $c$ avoiding $L$.

When $c$ corresponds to a geodesic boundary, the total mass of $c$ does not give information about the orientation of spiraling of leaves around $c$. If we choose for each boundary component a positive way of spiraling, then we can define a signed mass of $\bar{m}(c)$ in the following way:

- $|\bar{m}(c)|=m(c)$;

- $\bar{m}(c)>0$ if and only if it spirals in the positive way around $c$.

(The second requirement makes sense because two leaves near $c$ have to spiral in the same way.)

Let us stress that the signed mass of $c$ can be defined only for punctures corresponding to geodesic boundary components, and it is well defined up to the choice of a positive way of spiraling.

\subsection{Geodesic laminations on a pair of pants}

Here we give an explicit description of the measured geodesic laminations on a hyperbolic pair of pants in terms of the signed masses. This case is relevant to what in the physics literature is known as the 3 asymptotic region black hole (see [3, 4]). 
Proposition 2.4. Fix a hyperbolic pair of pants $P$, and for each boundary component choose a spiraling orientation. Then the function that associates to every measured geodesic lamination on $P$ the signed masses of the boundary components of $P$ is bijective.

Proof. Denote by $c_{1}, c_{2}, c_{3}$ both the punctures of $\Sigma_{0,3}$ and the corresponding boundary curves on $P$.

Since simple closed curves in $\Sigma_{0,3}$ are boundary parallel, geodesic laminations do not contain a compact part. Moreover, there are six properly embedded arcs up to homotopy. Each of them is determined by its endpoints. There are three arcs connecting different punctures and three arcs connecting the same puncture (see [12]).

Thus, there exist exactly four maximal systems of disjoint properly embedded $\operatorname{arcs}$ in $\Sigma_{0,3}$, each with exactly 3 arcs. Namely, $L_{0}$ is the union of arcs connecting different components whereas $L_{i}$ (for $i=1,2,3$ ) is the union of arcs with endpoint at $c_{i}$ : one with both endpoints at $c_{i}$, the other two with one endpoint at $c_{i}$ and one at each of the other boundary components. There are also some non-maximal systems of disjoint properly embedded arcs, obtained by removing one, two or three arcs from a maximal system.

If we choose a way of spiraling around each boundary component and positive weights on segments of some $L_{i}$, these data uniquely determine a measured geodesic lamination on $P$, according to Lemma 2.3.

The mass of each boundary component $c$ is equal to the sum of the weights of the segments of $L_{i}$ ending at $c$, whereas the sign of $\bar{m}\left(c_{i}\right)$ is positive or negative depending on the chosen way of spiraling. So, in order to prove the statement, it is sufficient to show that given three positive numbers $m_{1}, m_{2}, m_{3}$, exactly one system of disjoint properly embedded arcs can be equipped with a system of positive weights which give masses equal to $m_{i}$. Generically, this system will be maximal (and therefore one of the $L_{i}, 0 \leq$ $i \leq 3$ ) but for some non-generic values of the $m_{i}$ it might be non-maximal, so that it will correspond to several of the $L_{i}$, each time with a weight 0 on one of the arcs.

Let $a, b, c$ be a system of weights on segments of $L_{0}$ as in Figure 3 . The signed masses of boundary curves are, respectively, $m_{1}=a+c, m_{2}=a+b$, and $m_{3}=b+c$. Notice that in this case the $m_{i}$ are all positive and satisfy the triangle inequality $m_{i} \leq m_{j}+m_{k}$. Moreover, the weights $a, b, c$ are explicitly determined by $m_{1}, m_{2}, m_{3}$. Indeed, we have

$$
a=\frac{m_{1}+m_{2}-m_{3}}{2} \quad b=\frac{m_{2}+m_{3}-m_{1}}{2} \quad c=\frac{m_{1}+m_{3}-m_{2}}{2}
$$



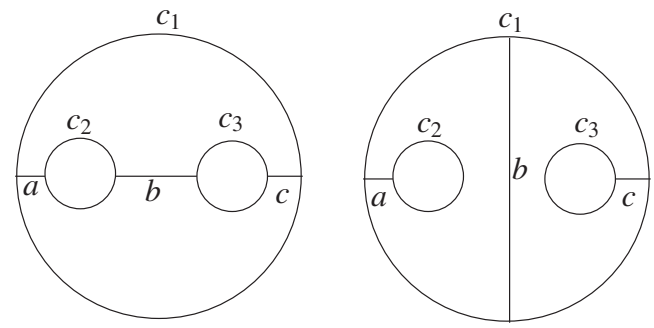

Fig. 3. On the left the system $L_{0}$ and on the right the system $L_{1}$.

Let's now consider a system of weights $a, b, c$ on $L_{1}$ as in Figure 3. The corresponding masses in this case are $m_{1}=a+c+2 b, m_{2}=a$, and $m_{3}=c$. Notice that in this case $m_{1} \geq m_{2}+m_{3}$. Moreover, $m_{1}, m_{2}, m_{3}$ determine explicitly the weights $a, b, c$. Namely

$$
a=m_{2}, \quad b=\frac{m_{1}-m_{2}-m_{3}}{2}, \quad c=m_{3} .
$$

Given three generic positive numbers $m_{1}, m_{2}, m_{3}$, this computation shows that exactly one of the $L_{i}, 0 \leq i \leq 3$, can be equipped with a system of weights which gives masses equal to $m_{i}$. The system of weights is uniquely determined as well. If now the $m_{i}$ satisfy the equality in one of the triangle inequalities (it is non-generic), then more than one of the $L_{i}$ is acceptable, but each time with one of the weights equal to zero.

In particular, the measures on $L_{0}$ correspond to $m_{1}, m_{2}, m_{3}$ satisfying the three triangle inequalities, whereas the measures on $L_{i}$ correspond to the case $m_{i} \geq m_{j}+$ $m_{k}$.

\section{Earthquakes}

In this section, we recall the definition of earthquakes on hyperbolic surfaces, in a way which is adapted to hyperbolic surfaces with geodesic boundary, and show how the definition can be extended to this setting.

\subsection{Earthquakes on convex subsets of $\mathbb{H}^{2}$ with geodesic boundary}

Let $\mathcal{H}$ be an open convex set with geodesic boundary in $\mathbb{H}^{2}$ and $L$ be a geodesic lamination of $\mathcal{H}$. By definition, a stratum of $L$ is either a leaf of $L$ or a component of $\mathcal{H} \backslash L$. A right earthquake on $\mathcal{H}$ with fault locus $L$ is a (possibly discontinuous) map

$$
E: \mathcal{H} \rightarrow \mathbb{H}^{2}
$$


with the property that

- for every stratum $F$, there is an isometry $A(F) \in P S L_{2}(\mathbb{R})$ such that $\left.E\right|_{F}=$ $\left.A(F)\right|_{F}$,

- given two strata $F$ and $F^{\prime}$ the comparison map $A(F)^{-1} \circ A\left(F^{\prime}\right)$ is a hyperbolic transformation whose axis weakly separates $F$ from $F^{\prime}$ and translates $F^{\prime}$ to the right as seen from $F$.

Given an earthquake on $\mathcal{H}$ with fault locus $L$, we can equip $L$ with a transverse measure that encodes the amount of shearing. More precisely, given a path $c:[0,1] \rightarrow \mathcal{H}$ transverse to $L$ and given a partition $I=\left(0=t_{0}<t_{1}<\cdots<t_{k}=1\right)$ we consider the number $\mu(C ; I)$ that is the sum of the translation lengths of the comparison maps $A\left(F\left(t_{i+1}\right) A\left(F\left(t_{i}\right)\right)^{-1}\right.$ where $F(t)$ is the stratum through $c(t)$.

By a standard fact of hyperbolic geometry on the composition of hyperbolic transformations with disjoint axes, if $I^{\prime}$ is finer than $I$ then $\mu\left(c ; I^{\prime}\right) \leq \mu(c ; I)$. Thus, we can define

$$
\mu(c)=\inf _{I} \mu(C ; I)=\lim _{|I| \rightarrow 0} \mu(C ; I),
$$

and $\mu$ defines a transverse measure on $L$.

Thurston showed that the measured lamination $\lambda=(L, \mu)$ determines the earthquake $E[20]$.

Proposition 3.1. Given a measured geodesic lamination $\lambda$ on $\mathcal{H}$, there is a unique earthquake (up to post-composition with isometries of $\mathbb{H}^{2}$ ) with shearing lamination $\lambda$.

Contrary to the case discussed in [20] where earthquakes are bijective maps from $\mathbb{H}^{2}$ to itself, in our setting the image of the earthquake does not need to be the whole $\mathbb{H}^{2}$. This is the reason why Proposition 3.1 holds in our setting whereas it was not true in [20].

On the other hand, it is not difficult to prove that for every earthquake $E: \mathcal{H} \rightarrow$ $\mathbb{H}^{2}$ the image $E(\mathcal{H})$ is a convex set with geodesic boundary because it is a connected union of geodesics and ideal hyperbolic polygons (see Lemma 8.4). 


\subsection{Earthquakes on $\mathcal{T}_{\boldsymbol{g}, \mathbf{n}}$}

Given an admissible hyperbolic metric $\eta$ on $\Sigma$, the left and right earthquakes along a measured geodesic lamination $\lambda$ can be defined like in the compact case.

When the lamination is locally finite they can be described in a very simple way. The right earthquake along $\lambda$ is obtained by shearing each component of $\Sigma \backslash \lambda$ to the right of the adjacent component by a factor equal to the mass of the boundary.

For the general case, it is convenient to construct an equivariant earthquake on the universal covering.

The universal covering of $\Sigma_{\eta}$, say $\mathcal{H}$, is an open convex subset with geodesic boundary in $\mathbb{H}^{2}$. More precisely, $\mathcal{H}$ is the convex hull of the limit set of the holonomy $h$ of $\eta$.

The lifting of $\lambda$ is a $h$-invariant measured geodesic lamination $\tilde{\lambda}$. Consider the right earthquake along $\tilde{\lambda}$, say

$$
E: \mathcal{H} \rightarrow \mathbb{H}^{2}
$$

By the invariance of $\tilde{\lambda}$, it turns out that $E \circ h(\gamma)$ is still an earthquake with shearing lamination $\lambda$.

By Proposition 3.1, for every $\gamma \in \pi_{1}(\Sigma)$ there is an element $h^{\prime}(\gamma) \in P S L_{2}(\mathbb{R})$ such that

$$
E \circ h(\gamma)=h^{\prime}(\gamma) \circ E
$$

Proposition 3.2. The representation $h^{\prime}$ is faithful and discrete. The quotient $\mathbb{H}^{2} / h^{\prime}$ is homeomorphic to $\Sigma$. The map $E$ induces to the quotient a piecewise isometry

$$
E_{\lambda}^{r}: \Sigma_{\eta} \rightarrow E(\mathcal{H}) / h^{\prime}
$$

The surface $E(\mathcal{H}) / h^{\prime}$ coincides with the convex core of $\mathbb{H}^{2} / h^{\prime}$ (it is in particular an admissible surface).

Proof. First, notice that $h^{\prime}$ is discrete. Indeed, let $p$ be some point contained in the interior of some two-dimensional stratum $F$ of $\tilde{\lambda}$. Now the $h^{\prime}$-orbit of $E(p)$ accumulates at $E(p)$ if and only if the $h$-orbit of $p$ accumulates at $p$. This shows that the orbit of $E(p)$ is discrete. Thus, $h^{\prime}$ is a discrete representation. Since the earthquake map is injective, it turns out that $h^{\prime}$ is faithful. 
To prove that $\mathbb{H}^{2} / h^{\prime} \cong \Sigma$, notice that $h$ and $h^{\prime}$ are connected by a path of faithful and discrete representations. Namely, let $h_{t}$ be the representation corresponding to the earthquake along $t \lambda$.

To conclude the proof, we have to check that $E(\mathcal{H})$ is the convex hull of the limit set of $h^{\prime}$. By [5] $E(\mathcal{H})$ is a convex set with geodesic boundary. Let $\tilde{U}$ be the lifting on $\mathcal{H}$ of a regular neighborhood of punctures in $\Sigma_{\eta}$. A simple argument shows that $\mathcal{H} \backslash \tilde{U}$ is sent by $E$ to a subset with compact quotient.

Thus, it is sufficient to show that there is a constant $M$, such that for any point $p$ close to a puncture $x$ there exists a loop centered at $p$, parallel to the puncture, whose length is bounded by $M$.

Take the geodesic loop $\gamma$ of $\Sigma_{\eta}$ centered at $p$ and parallel to $x$. Notice that $\gamma$ meets only a finite number of leaves of $L$.

The image of $\gamma$ via $E_{\lambda}^{r}$ is a union of geodesic arcs $\gamma_{i}$ whose endpoints $x_{i}, y_{i}$ lie on $E_{\lambda}^{r}(L)$. The piecewise geodesic loop

$$
\hat{\gamma}=\gamma_{0} *\left[y_{0}, x_{1}\right] * \gamma_{1} *\left[y_{1}, x_{2}\right] * \ldots * \gamma_{N}
$$

is parallel to $x$. Notice that the sum of the lengths of $\gamma_{i}$ is equal to the length of $\gamma$, whereas the length of the segment $\left[y_{i}, x_{i+1}\right]$ is equal to the mass of the corresponding leaf. Thus, the length of $\hat{\gamma}$ is equal to the sum of the length and the mass of $\gamma$.

We say that $E(\mathcal{H}) / h^{\prime}$ is obtained by a right earthquake of $\Sigma_{\eta}$ along $\lambda$ and we denote it by $E_{\lambda}^{r}\left(\Sigma_{\eta}\right)$.

We have seen that a lamination on $\Sigma_{\eta}$ is the disjoint union of a compact part, say $\lambda_{c}$, and a finite union of leaves that spiral around boundary components or enter cusps, say $\lambda_{b}$. The earthquake along $\lambda$ can be regarded as the composition of the earthquake along $\lambda_{c}$ and the earthquake along $\lambda_{b}$ : more precisely we have to compose the earthquake along $\lambda_{c}$ with the earthquake along $\hat{\lambda}_{b}$ that is the image of $\lambda_{b}$ in $E_{\lambda_{c}}^{r}\left(\Sigma_{\eta}\right)$.

The earthquake along $\lambda_{c}$ can be easily understood: we approximate $\lambda_{c}$ by weighted multicurves. Then the earthquake along $\lambda_{C}$ is the limit of the fractional Dehn twists along these weighted multicurves. Notice that the earthquake along $\lambda_{C}$ does not change the length of any boundary component.

The earthquake along $\lambda_{b}$ can be described in the following way. We cut the surface (only the interior of $\Sigma_{\eta}$ ) along the leaves of $\lambda_{b}$ and we get a surface $\hat{\Sigma}$ with geodesic boundary. Since $\lambda_{b}$ is locally finite in $\Sigma_{\eta}$, every leaf of $\lambda_{b}$ corresponds to exactly two boundary components of $\hat{\Sigma}$. Then we glue back the boundary components correspond- 
ing to the same leaf $l$, composing the original gluing with a right translation of factor equal to the weight of $l$.

Opposite to the previous case, the earthquake along $\lambda_{b}$ changes the length of the boundary components (and may transforms cusps in geodesic boundary components). In the next section, we determine the length of a boundary component after the earthquake.

\subsection{Boundary length and spiraling orientation after an earthquake}

The mass of a boundary component $c$ for a measured lamination $\lambda$ is in direct relation with the variation of the length of $c$ under an earthquake along $\lambda$, and also with the way $\lambda$ spirals on $c$. Indeed, the image $\lambda^{\prime}$ of $\lambda$ by the right earthquake $E_{\lambda}^{r}$ is well defined, but it might spiral on $c$ differently from $\lambda$.

Let us choose an explicit way of spiraling around each boundary curve in the following way. An orientation is induced by $P$ on its boundary. If $l$ spirals around some $c_{i}$, then an orientation is induced on $l$ by the orientation on $c_{i}$. Namely, $l_{i}$ is oriented in such a way that the nearest-point retraction on $c_{i}$ (i.e., well defined in a neighborhood of $c_{i}$ ) is orientation preserving. Notice that if $l$ spirals around $c_{i}$ and $c_{j}$ the orientations induced on $l$ may disagree.

Then we say that $l$ spirals in a positive way around $c_{i}$ if it goes closer and closer to $l$. We call it the standard spiraling orientation, and we will refer to it throughout this paper.

Proposition 3.3. Let $a$ be the length of $c$ in $\Sigma_{\eta}$, and let $a^{\prime}$ be the length of the corresponding boundary component after a left earthquake along $\lambda$, in $E_{\lambda}^{r}\left(\Sigma_{\eta}\right)$.

(1) $a^{\prime}=a+m$ if $\lambda$ spirals around $c$ in the positive way, $a^{\prime}=|a-m|$ if $\lambda$ spirals around $c$ in the negative way.

(2) If $\lambda$ spirals in the positive direction, so does $\lambda^{\prime}$. If $\lambda$ spirals in the negative direction, then $\lambda^{\prime}$ spirals in the negative direction if $m<a$, in the positive direction if $m>a$.

(3) $E_{\lambda}^{r}\left(\Sigma_{\eta}\right)$ has a cusp at the boundary component corresponding to $c$ if and only if $\lambda$ spirals in the negative direction at $c$ and its mass $m$ is equal to the length $a$ of $c$ in $\eta$. 


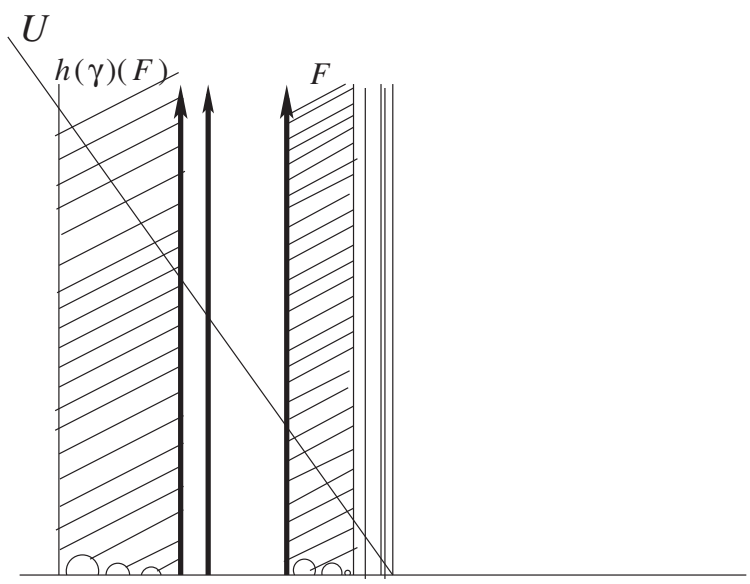

Fig. 4. Proof of Proposition 3.3.

Proof. Let us consider the lifting of $E_{\lambda}^{r}$ to the universal covering

$$
E: \mathcal{H} \rightarrow \mathcal{H}^{\prime}
$$

Let $\tilde{c}$ be a lifting of $c$. In the upper-plane model of $\mathbb{H}^{2}$, we can suppose that $\tilde{c}$ is the geodesic from 0 to $\infty$ and $\mathcal{H}$ is contained in the region $\{(x, y) \mid x<0, y>0\}$.

Suppose that $\lambda$ spirals in the positive way around $c$. This means that there is an $\epsilon$-neighborhood $U$ of $\tilde{c}$ such that every leaf intersecting $U$ goes to $\infty$.

Let $\gamma \in \pi_{1}(\Sigma)$ be a positive representative of the peripheral loop around $c$. Then $h(\gamma)$ is of the following form: $\left(\begin{array}{cc}e^{a} & 0 \\ 0 & e^{-a}\end{array}\right)$.

Fix a stratum $F$ intersecting $U$, and notice that $\infty$ is an ideal endpoint of $F$. It follows that $F$ and $h(\gamma)(F)$ share the ideal endpoint $\infty$. In particular, $\infty$ is an endpoint of all the leaves of $\tilde{\lambda}$ separating $F$ from $h(\gamma)(F)$. This implies that there are a finite number of such leaves $l_{1}, l_{2}, \ldots, l_{k}$ and the comparison isometry between $F$ and $h(\gamma)(F)$ is the composition of hyperbolic translations along the $l_{i}$ with attractive fixed points equal to $\infty$ and translation lengths equal to the weight $w_{i}$ of $l_{i}$ (see Figure 4).

Such translations are all of the form $\left(\begin{array}{cc}e^{w_{i}} & * \\ 0 & e^{-w_{i}}\end{array}\right)$, so their composition is a matrix of the form $\left(\begin{array}{cc}e^{\sum w_{i}} & * \\ 0 & e^{-\left(\sum w_{i}\right)}\end{array}\right)$. Since the sum of the weights of $l_{1}, \ldots, l_{k}$ is the mass of $c$, the translation length of the comparison isometry is $m$. 
Since $h^{\prime}(\gamma)$ is the composition of the comparison isometry with $h(\gamma)$, this shows that the translation length of $h^{\prime}(\gamma)$ is $a+m$.

Moreover, notice that $\infty$ is the attractive fixed point of $h^{\prime}(\gamma)$ and that $E_{\lambda}^{r}(l)$ ends at $\infty$ for every leaf $l$ that ends at $\infty$. This show that the image lamination spirals in the positive way around $c$.

The other cases can be obtained by suitable adaptations of the same arguments.

The computation of the proof of Proposition 3.3 can also be found in [19] in the special case of a pair of pants, and in [7] in the slightly different setting of shear coordinates. The same proposition also holds—with positive and negative orientations reversed—for a left earthquake.

Earthquakes on a pair of pants. One could wonder whether the analog of Theorem 1.1 holds also for $\mathcal{T}_{g, n}$, that is whether, given $F, F^{\prime} \in \mathcal{T}_{g, n}$ there exists a unique $\lambda \in \mathcal{M} \mathcal{L}_{g, n}$ such that the left earthquake along $\lambda$ transforms $F$ into $F^{\prime}$. A classical example due to Thurston shows that this is not the case on a hyperbolic pair of pants. In this section, we will focus on that example. Since explicit computations are possible, we get a complete picture about earthquakes. In the next sections, we will see that the same picture, suitably expanded, holds for general surfaces.

Let $\Sigma$ be the thrice-punctured sphere and let $c_{1}, c_{2}, c_{3}$ denote the punctures. It is well known that a hyperbolic metric with geodesic boundary on $\Sigma$ is determined by three positive numbers $a_{1}, a_{2}, a_{3}$ corresponding to the lengths of the three boundary components. Moreover when $a_{i} \rightarrow 0$, the corresponding geodesic boundary component degenerates to a cusp. Thus, $\mathcal{T}_{0,3}$ is parameterized by a triple of non-negative numbers. Let $P\left(a_{1}, a_{2}, a_{3}\right)$ denote the element of $\mathcal{T}_{0,3}$ corresponding to the triple $\left(a_{1}, a_{2}, a_{3}\right)$.

We have seen in Proposition 2.4 that each measured geodesic lamination on $P$ is determined by three real numbers (the signed masses with respect to the standard spiraling orientation). Denote by $\lambda\left(m_{1}, m_{2}, m_{3}\right)$ the lamination corresponding to the triple $m_{1}, m_{2}, m_{3}$. Then the surface obtained by the right earthquake along $\lambda\left(m_{1}, m_{2}, m_{3}\right)$ on $P\left(a_{1}, a_{2}, a_{3}\right)$ is

$$
P\left(\left|a_{1}+m_{1}\right|,\left|a_{2}+m_{2}\right|,\left|a_{3}+m_{3}\right|\right)
$$

whereas the surface obtained by a left earthquake is

$$
P\left(\left|a_{1}-m_{1}\right|,\left|a_{2}-m_{2}\right|,\left|a_{3}-m_{3}\right|\right) .
$$




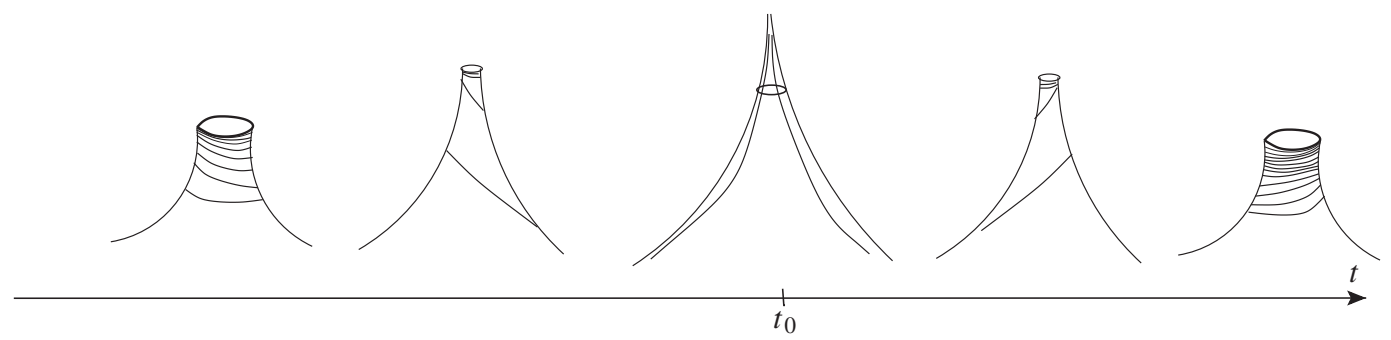

Fig. 5. Unwinding a boundary component into a cusp, and beyond.

Notice that these formulas make sense also when some $a_{i}=0$. In fact, in such a case they depend only on $\left|m_{i}\right|$ (we have previously remarked that it is not possible to define a signed mass corresponding to a cusp).

It follows from those formulas that two hyperbolic pairs of pants (without cusps) are related by eight earthquakes. In fact, for each $i$ we can choose arbitrarily the corresponding sign of $m_{i}$.

Let us focus on some points.

(1) Given two hyperbolic pairs of pants $P_{0}=P\left(a_{1}, a_{2}, a_{3}\right)$ and $P_{1}=P\left(b_{1}, b_{2}, b_{3}\right)$ there exists a unique lamination $\lambda$ such that $E_{\lambda}^{r}\left(P_{0}\right)=P_{1}$ and the path $E_{t \lambda}^{r}\left(P_{0}\right)$ is contained in the interior of $\mathcal{T}_{3,0}$, for $t \in[0,1]$. Namely, $\lambda=\lambda\left(b_{1}-a_{1}, b_{2}-\right.$ $\left.a_{2}, b_{3}-a_{3}\right)$.

(2) Take a measured geodesic lamination $\lambda=\lambda\left(m_{1}, m_{2}, m_{3}\right)$ and suppose $m_{1}<$ $-a_{1}$. Consider the earthquake path

$$
P_{t}=E_{t \lambda}^{r} P\left(a_{1}, a_{2}, a_{3}\right) \quad \lambda_{t}=\mathcal{E}_{t \lambda}(\lambda)
$$

for $t \in[0,1]$. It has a critical value at $t_{0}=-m_{1} / a_{1}$ where the length of $c_{1}$ becomes 0 , that means that $c_{1}$ becomes a cusp (see Figure 5).

Let us give a picture of the behavior of $P_{t}$ near $t_{0}$. For $t=t_{0}-\varepsilon$, the geodesic boundary $c_{1}$ is very small and by consequence there is a "big" regular neighborhood $U$ (i.e., the distance of $\partial U$ from $c_{1}$ is big). The geodesic lamination spirals in the positive direction, but it looks almost unwind. At time $t_{0}$, the geodesic boundary has disappeared and we have a cusp. The geodesic lamination is completely unwinded. As $t$ becomes greater than $t_{0}, c_{1}$ turns out to be a geodesic boundary component, but this time the geodesic lamination spirals in the opposite direction. 
(3) Let $\hat{\mathcal{T}}_{3,0}$ be the space of admissible hyperbolic structures on $\Sigma$ equipped with a positive spiraling orientation on each boundary component, that is, the enhanced Teichmüller space of the thrice-punctured sphere. Notice that this space could be identified with $\mathbb{R}^{3}$. In fact, each such surface is determined by three non-negative numbers (the lengths) and a certain number of "signs" corresponding to non-zero numbers. $\mathcal{T}_{3,0}$ could be regarded as the quotient of $\hat{\mathcal{T}}_{3,0}$ by the action of the group $G=\left(\mathbb{Z}^{2}\right)^{3}$, generated by the symmetries along coordinate planes.

(4) Take an element $P_{0}=P\left(a_{0}, a_{1}, a_{2}\right) \in \hat{\mathcal{T}}_{3,0}$. Recall that $P_{0}$ is a hyperbolic pant equipped with a spiraling orientation on each boundary component. The length of $c_{i}$ is $\left|a_{i}\right|$ whereas the spiraling orientation at $c_{i}$ agrees with the standard orientation depending on the sign of $a_{i}$.

Given a measured geodesic lamination $\lambda$ on $P_{0}$, we consider the signed masses with respect to the spiraling orientation of $P_{0}$-say $m_{1}, m_{2}, m_{3}-$ as well as the signed masses with respect to the standard spiraling orientation-say $\hat{m}_{1}, \hat{m}_{2}, \hat{m}_{3}$.

By the choice of the sign of $a_{i}$, we clearly have

$$
\hat{m}_{i}=\operatorname{sign}\left(a_{i}\right) m_{i}
$$

(5) Suppose all $a_{i} \neq 0$ (i.e., $P_{0}$ is a pair of pants). Then a spiraling orientation can be pushed forward on the surface $E_{\lambda}^{r}\left(P_{0}\right)$ in the following way.

If no leaf of $\lambda$ spirals around $c_{i}$, then $E_{\lambda}^{r}$ restricts to an isometry of some neighborhood of $c_{i}$ in $P_{0}$ on to some neighborhood of $c_{i}$ in $E_{\lambda}^{r}\left(P_{0}\right)$, so we can consider the orientation induced by the earthquake map.

If a leaf $l$ of $\lambda$ spirals around $c_{i}$, the image through $E_{\lambda}^{r}$ of $l$ is still a geodesic $l^{*}$ spiraling around $c_{i}$. In this case, we choose the spiraling orientation around $c_{i}$, in such a way that $l^{*}$ spirals in a positive direction iff $l$ does in $P_{0}$. Thus, earthquakes "lift" to a map

$$
\mathcal{E}_{\lambda}: \hat{\mathcal{T}}_{3,0} \backslash\{\text { structures with cusp }\} \rightarrow \hat{\mathcal{T}}_{3,0}
$$

If $b_{1}, b_{2}, b_{3}$ are the real parameters corresponding to $\mathcal{E}_{\lambda}\left(P_{0}\right)$, by Proposition 3.3 we have

$$
\left|b_{i}\right|=|| a_{i}\left|+\hat{m}_{i}\right|=\left|a_{i}+m_{i}\right|
$$


where $\hat{m}_{i}$ is the signed mass of $\lambda$ with respect to the standard spiraling orientation and $m_{i}$ is the signed mass with respect to the spiraling orientation of $P_{0}$.

About the sign of $b_{i}$, notice that if $m_{i}^{*}$ denotes the signed mass around $c_{i}$ of $E_{\lambda}^{r}(\lambda)$ with respect to the spiraling orientation of $\mathcal{E}_{\lambda}\left(P_{0}\right)$ we have $m_{i}^{*}=m_{i}$.

On the other hand by Proposition 3.3, we have that the signed mass with respect to the standard spiraling orientation is given by $\hat{m}_{i}^{*}=\left(\operatorname{sign}\left|a_{i}\right|+\hat{m}_{i}\right) \hat{m}_{i}$. Since $m_{i}^{*}=\operatorname{sign}\left(b_{i}\right) \hat{m}_{i}^{*}$ and $m_{i}=\operatorname{sign}\left(a_{i}\right) \hat{m}_{i}$, we conclude that

$$
\operatorname{sign}\left(b_{i}\right)=\operatorname{sign}\left(a_{i}\right) \operatorname{sign}\left(\left|a_{i}\right|+\hat{m}_{i}\right)=\operatorname{sign}\left(a_{i}+m_{i}\right)
$$

So we get the simple formula

$$
b_{i}=a_{i}+m_{i}
$$

In particular, $\mathcal{E}_{\lambda}$ extends on the whole of $\hat{\mathcal{T}}_{0,3}$. Notice that if $P_{0}$ has a cusp in $c_{1}$, then the orientation of $\mathcal{E}_{\lambda}\left(P_{0}\right)$ in $c_{1}$ depends only on the sign of $m_{i}$.

(6) $\mathcal{E}_{\lambda}$ is not $G$-equivariant on $\hat{\mathcal{T}}_{3,0}$. On the other hand, it is uniquely determined by the following conditions:

- if $\mathcal{T}_{3,0}$ is identified with the subset of $\hat{\mathcal{T}}_{3,0}$ corresponding to triples $\left(a_{1}, a_{2}, a_{3}\right)$ with $a_{k} \geq 0$, then $\pi \circ \mathcal{E}_{\lambda}=E_{\lambda}^{r}$ (where $\pi: \hat{\mathcal{T}}_{3,0} \rightarrow \mathcal{T}_{3,0}$ is the projection).

- $\mathcal{E}_{\lambda}$ is a flow, that is $\mathcal{E}_{t \lambda} \circ \mathcal{E}_{t^{\prime} \lambda}=\mathcal{E}_{\left(t+t^{\prime}\right) \lambda}$ for $t, t^{\prime}>0$.

(7) On $\hat{\mathcal{T}}_{0,3}$, the Earthquake Theorem holds. That is, there exists a unique right earthquake joining two points in $\hat{\mathcal{T}}_{3,0}$.

From pairs of pants to general surfaces. We can summarize the previous remarks as follows. Even if the Earthquake Theorem does not hold for $\mathcal{T}_{3,0}$, we have seen that $\mathcal{T}_{3,0}$ can be regarded as the quotient of a bigger smooth space $\hat{\mathcal{T}}_{3,0}$ (defined by keeping track of spiraling orientations) by the action of $\left(\mathbb{Z}_{2}\right)^{3}$. The earthquakes lift to equivariant maps of $\hat{\mathcal{T}}_{3,0}$ and the Earthquake Theorem holds for the space $\hat{\mathcal{T}}_{3,0}$.

In the next sections, we will see that the same picture, suitably extended, holds for any surface $\Sigma_{g, n}$. However, measured geodesic laminations on general surfaces are more complicated because they are the sum of a compact part (i.e., in general not locally finite) and a non-compact locally finite part. A simple doubling argument shows that if $S$ and $S^{\prime}$ are homeomorphic hyperbolic surfaces such that the lengths of the corresponding boundary curves are equal then there is a unique compact lamination $\lambda$ on $S$ such that $E_{\lambda}^{r}(S)=S^{\prime}$. On the other hand, earthquakes that modify the lengths of the boundary 
curves contain leaves spiraling around boundary curves. So one could wonder whether it is possible to construct the earthquake between $S$ and $S^{\prime}$ by looking separately the compact part and the non-compact part of the lamination.

But the earthquake along a lamination without compact part changes the metric in the interior of the surface in a non-trivial way. Moreover, the topology of the noncompact part of the lamination imposes topological constraints on the non-compact part. The interesting aspect of Theorem 1.2 rests in this somewhat subtle interplay between the compact and the non-compact parts of the lamination.

The key ingredient to get such a generalization is to relate earthquakes to bent surfaces in the multi-black holes that are defined in the next section. The relation between earthquakes and bent surfaces will be obtained by generalizing the Mess argument in the closed case. The main difference will be that in a multi-black hole there are (finitely) many bent surfaces (in contrast in the closed case where there is a unique one).

\section{The Geometry of Anti-de Sitter Space}

We collect in this section, for the reader's convenience, some basic facts on the geometry of the three-dimensional AdS space, as can be found in particular in [17, 2].

The AdS space and its conformal boundary. Let $\mathbb{R}^{2,2}$ denote $\mathbb{R}^{4}$ equipped with the standard bilinear symmetric form, say $\langle\cdot, \cdot\rangle$, of signature $(2,2)$.

Let us consider the set of negative unit vectors:

$$
X:=\left\{x \in \mathbb{R}^{2,2} \mid\langle x, x\rangle=-1\right\} .
$$

Since the tangent plane at $x$ of $X$ is the linear plane orthogonal to $x$ with respect to $\langle\cdot, \cdot\rangle$, the induced symmetric form on $X$ has Lorentzian signature.

The projection

$$
\pi: X \rightarrow \mathbb{R} P^{3}
$$

is a $2: 1$ covering on its image. By definition, the (projective model of) Anti-de Sitter space is the image of $X$ :

$$
\mathrm{AdS}_{3}:=\pi(X)=\left\{[x] \in \mathbb{R} P^{3} \mid\langle x, X\rangle<0\right\} .
$$

Since the covering transformation of $\pi$ preserves the metric, a Lorentzian metric is defined on $\mathrm{AdS}_{3}$. It is a geodesically complete Lorentzian manifold of constant curvature 
-1. (Some authors define the AdS space as the double cover of the $\mathrm{AdS}_{3}$ space defined here, but this only introduces minor differences in the notations.)

Notice that $\mathrm{AdS}_{3}$ is an open domain in $\mathbb{R} P^{3}$ whose boundary is the projective quadric

$$
\partial_{\infty} \mathrm{AdS}_{3}:=\left\{x \in \mathbb{R} P^{3} \mid\langle x, x\rangle=0\right\}
$$

This quadric is a doubly ruled surface: this precisely means that there are two foliations $\mathcal{F}_{l}$ and $\mathcal{F}_{r}$ on $\partial_{\infty} \mathrm{AdS}_{3}$ whose leaves are projective lines and such that the intersection of a leaf $l \in \mathcal{F}_{l}$ with a leaf $l^{\prime} \in \mathcal{F}_{r}$ is exactly one point.

Topologically $\partial_{\infty} \mathrm{AdS}_{3}$ is a torus and it disconnects $\mathbb{R} P^{3}$ in two solid tori. It is possible to orient the leaves of each foliation $\mathcal{F}_{l}$ and $\mathcal{F}_{r}$ in such a way that if $c_{l}$ and $c_{r}$ denote, respectively, the homology classes of the oriented leaves of the two foliations then the meridian corresponding to $\mathrm{AdS}_{3}$ is homologous to $\pm\left(c_{l}+c_{r}\right)$ and the meridian corresponding to the complement of $\mathrm{AdS}_{3}$ is homologous $\pm\left(c_{r}-c_{l}\right)$. There are two possible way to choose such orientations. We fix arbitrary one of these choices. We consider on the boundary of $\mathrm{AdS}_{3}$ the orientation such that if $e_{1}$ is a positive vector tangent to the left foliation at $p$ and $e_{\mathrm{r}}$ is the positive vector tangent to the right foliation then $\left(e_{1}, e_{\mathrm{r}}\right)$ is a positive basis of $T_{p} \partial_{\infty} \mathrm{AdS}_{3}$. Moreover, we consider on $\mathrm{AdS}_{3}$ the orientation that is compatible with the orientation of the boundary.

The space $\mathrm{AdS}_{3}$ is not simply connected. Nevertheless, isometries act transitively on the orthonormal frames. This implies that every Lorentzian manifold of constant curvature -1 is equipped with a ( $\left.\operatorname{Isom}_{0}, \mathrm{AdS}_{3}\right)$ structure.

Geodesics in $\mathrm{AdS}_{3}$ are projective lines. There is a fairly simple way to distinguish time-like from space-like geodesics. In fact, time-like geodesics correspond to projective lines entirely contained in $\mathrm{AdS}_{3}$. They are closed simple lines of length $\pi$. Light-like lines correspond to projective lines that are tangent to the boundary. Finally, space-like lines correspond to projective lines that meet the boundary in two different points. They are open geodesics of infinite length.

As a consequence, totally geodesic planes are obtained by intersecting $\mathrm{AdS}_{3}$ with projective planes. Still in this case, there is a topological way to distinguish space-like planes from time-like and light-like planes. Indeed, light-like planes correspond to projective planes tangent to $\partial_{\infty} \mathrm{AdS}_{3}$ (that intersects the boundary along two leaves). Timelike planes are topologically Moebius bands (they cut $\partial_{\infty} \mathrm{AdS}_{3}$ along a meridian of the exterior of $\mathrm{AdS}_{3}$ ). Finally, space-like planes are compression disks (and they cut $\partial_{\infty} \mathrm{AdS}_{3}$ along a meridian of $\mathrm{AdS}_{3}$ ). 
Notice that any space-like plane $P$ can be oriented by requiring that its trace at infinity with the induced orientation is homologous to $C_{l}+c_{r}$. This is called the positive orientation of $P$. We fix the following time orientation on $\mathrm{AdS}_{3}$ : a time-like vector $v$ at some point $p \in \mathrm{AdS}_{3}$ is future pointing, if it induces on the space-like plane $P$ through $p$ orthogonal to $v$ the positive orientation.

Intrinsically, a space-like plane is isometric to $\mathbb{H}^{2}$. Indeed, it is a simply connected geodesically complete surface of constant curvature -1 . Moreover, it can be shown that every isometry between $\mathbb{H}^{2}$ and a plane $P_{0}$ extends to a projective map

$$
r: \mathbb{R} P^{2} \rightarrow \mathbb{R} P^{3}
$$

(where we are using the projective model of $\mathbb{H}^{2}$ ).

In particular, $r$ identifies $\partial \mathbb{H}^{2}$ with $\partial_{\infty} P_{0}$. We can consider the maps

$$
\partial \mathbb{H}^{2} \rightarrow \mathcal{F}_{l} \quad \partial \mathbb{H}^{2} \rightarrow \mathcal{F}_{r}
$$

that associates to a point $p \in \partial \mathbb{H}^{2}=\partial_{\infty} P_{0}$ the left and the right leaves through it. By transversality both maps are local homeomorphisms and for homological reasons they have degree one, so these maps are homeomorphisms. This precisely means that every leaf of $\mathcal{F}_{l}$ (resp. $\mathcal{F}_{r}$ ) meets $\partial \mathbb{H}^{2}$ exactly in one point.

We fix once and for all an isometric totally geodesic embedding

$$
r_{0}: \mathbb{H}^{2} \rightarrow P_{0}
$$

and we consider the induced identification $\partial_{\infty} \mathrm{AdS}_{3}$ and $\partial \mathbb{H}^{2} \times \partial \mathbb{H}^{2}$. Namely, any point $p \in$ $\partial_{\infty} A d S_{3}$ is identified to the pair $\left(x_{l}(p), x_{r}(p)\right)$ where $x_{l}(p)$ (resp. $\left.x_{r}(p)\right)$ is the intersection of the left (resp. right) leaf through $p$ with $\partial \mathbb{H}^{2}=\partial_{\infty} P_{0}$.

Since isometries of $\mathrm{AdS}_{3}$ are projective maps that leave $\partial_{\infty} \mathrm{AdS}_{3}$ invariant, then preserve the double ruling of $\partial_{\infty} \mathrm{AdS}_{3}$. In particular, the action of $\operatorname{Isom}_{0}$ on $\partial \mathbb{H}^{2} \times \partial \mathbb{H}^{2}$ is diagonal: for every $f \in \operatorname{Isom}_{0}$ we have $f(x, y)=\left(a_{l}(f)(x), a_{r}(f)(x)\right)$ where $a_{l}(f)$ and $a_{r}(f)$ are homeomorphisms of $\partial \mathbb{H}^{2}$. 
Lemma 4.1. [2] The maps $a_{l}(f)$ and $a_{r}(f)$ extends to isometries of $\mathbb{H}^{2}$. In particular, $a_{l}(f), a_{r}(f) \in P S L_{2}(\mathbb{R})$.

By this lemma, a homomorphism

$$
a: \operatorname{Isom}_{0} \ni f \mapsto\left(a_{l}(f), a_{r}(f)\right) \in P S L_{2}(\mathbb{R})^{2}
$$

is pointed out. If $a_{l}(f)=a_{r}(f)=I d$, it turns out that $f$ fixes $\partial_{\infty} \mathrm{AdS}_{3}$. Since $f$ is a projective map, it follows that $f=I d$. Thus, $a$ is injective. Since both $\operatorname{Isom}_{0}$ and $P S L_{2}(\mathbb{R})^{2}$ have dimension 6 , it follows that $a$ is also surjective, thus it is an isomorphism.

From now on, we use the map $a$ to state an identification between $P S L_{2}(\mathbb{R}) \times$ $\mathrm{PSL}_{2}(\mathbb{R})$ and $\mathrm{Isom}_{0}$.

Remark 4.2. The identification between $\partial_{\infty} \mathrm{AdS}_{3}$ with $\partial \mathbb{H}^{2} \times \partial \mathbb{H}^{2}$ is well defined once we fix a totally geodesic embedding $r_{0}: \mathbb{H}^{2} \rightarrow \mathrm{AdS}_{3}$.

The map $r_{0}$ is unique up to post-composition with isometries of $\mathrm{AdS}_{3}$. It follows that the identifications between $\partial_{\infty} \mathrm{AdS}_{3}$ and $\partial \mathbb{H}^{2} \times \partial \mathbb{H}^{2}$ and between Isom $_{0}$ and $P S L_{2}(\mathbb{R}) \times P S L_{2}(\mathbb{R})$ are uniquely determined up to isometries of $\mathrm{AdS}_{3}$.

Space-like planes are determined by their intersection with $\partial_{\infty} \mathrm{AdS}_{3}=\partial \mathbb{H}^{2} \times \partial \mathbb{H}^{2}$. By our description, it turns out that the trace at infinity of any space-like plane is the graph of some map $A \in P S L_{2}(\mathbb{R})$.

Indeed by definition, the trace at infinity of our fixed plane $P_{0}$ corresponds to the diagonal of $\partial \mathbb{H}^{2} \times \partial \mathbb{H}^{2}$. If $P$ is any other plane, there is an isometry $f$ of $\mathrm{AdS}_{3}$ such that $f\left(P_{0}\right)=P$. Thus by definition $\partial_{\infty} P=\left\{\left(a_{l}(f) x, a_{r}(f) x\right) \mid x \in \partial \mathbb{H}^{2}\right\}$. By setting $y=a_{l}(f) x$, we can also write

$$
\partial_{\infty} P=\left\{\left(y, a_{r}(f) a_{l}(f)^{-1} y\right) \mid y \in \partial \mathbb{H}^{2}\right\}
$$

that is, $\partial_{\infty} P$ is the graph of $a_{r}(f) a_{l}(f)^{-1}$.

Eventually, space-like planes are parameterized by elements in $P S L_{2}(\mathbb{R})$. Given $A \in P S L_{2}(\mathbb{R})$, we denote by $P_{A}$ the plane whose trace at infinity is the graph of $A$.

By this description, it is clear that given two planes $P, Q$ there is a unique $A$ in $P S L_{2}(\mathbb{R})$ such that $(1, A) \cdot P=Q$. Moreover, the stabilizer of every plane is conjugated to the diagonal subgroup into $P S L_{2}(\mathbb{R}) \times P S L_{2}(\mathbb{R})$. 
In what follows, we will also use the following

Lemma 4.3. The map

$$
\partial \mathbb{H}^{2} \ni x \mapsto(x, A x) \in \partial P_{A}
$$

extends uniquely to an isometry $r_{A}: \mathbb{H}^{2} \rightarrow P_{A}$.

Proof. It is sufficient to define $r_{A}=(1, A) \circ r_{0}$.

Bending angles between space-like planes. In Lorentzian geometry, there is a natural definition of angles between future-oriented time-like vectors. Indeed, the set of futureoriented unit time-like tangent vectors at a point $p$ of some Lorentzian manifold $X$, say $H_{p}$, is isometric to $\mathbb{H}^{2}$. If $v, w$ lie in $H_{p}$, we can define the angle between $v$ and $w$ as the distance in $H_{p}$ of $v$ and $w$. By a classical formula of hyperbolic geometry, it turns out that this angle is

$$
\cosh ^{-1}|\langle v, w\rangle|
$$

Notice that the definition is quite similar to the classical definition of angles in Riemannian geometry, the main difference being that the angle is a well-defined number in $[0,+\infty)$.

If $P$ and $Q$ are space-like totally geodesic planes in $\mathrm{AdS}_{3}$ meeting along a geodesic $l$, then their future-oriented unit normal vector fields are parallel along $l$. Thus, we can define the bending angle between $P$ and $Q$ as the angle between those vector fields.

If $l$ is oriented, we can also define a signed bending angle between $P, Q$. Indeed, given a point $p \in l$, let $v, u, w \in \operatorname{TAdS}_{3}$ be, respectively, the positive unit tangent vector along $l$, the future-pointing unit normal vector of $P$, and the future-pointing unit normal vector of $Q$. We say that the angle between $P$ and $Q$ is positive if the vectors $v, u, w$ form a positive basis of $\mathrm{AdS}_{3}$. It can be shown that the signed angle is

$$
\alpha(P, Q)=\sinh ^{-1} \omega_{p}(v, w, u)
$$

where $\omega$ is the volume form on $\mathrm{AdS}_{3}$. Notice that by definition the angle is skewsymmetric and it depends on the choice of the orientation of $l$.

The conformal structure on $\partial_{\infty} \mathrm{AdS}_{3}$. Let us identify $\partial_{\infty} \mathrm{AdS}_{3}$ with $\partial \mathbb{H}^{2} \times \partial \mathbb{H}^{2}$. If $\theta$ and $\phi$ denote positive-oriented parameters on each copy of $\partial \mathbb{H}^{2}$, then we can consider the 
Lorentzian metric $\eta=\mathrm{d} \theta \mathrm{d} \phi$ on $\partial_{\infty} \mathrm{AdS}_{3}$. The conformal class of $\eta$ is independent of the choice of coordinates and the group $P S L_{2}(\mathbb{R})^{2}$ acts conformally on $\partial \mathbb{H}^{2} \times \partial \mathbb{H}^{2}$.

Intrinsically, the conformal structure on $\partial_{\infty} \mathrm{AdS}_{3}$ is characterized by the fact that isotropic directions are tangent to the leaves of the double ruling on $\partial_{\infty} \mathrm{AdS}_{3}$. Indeed, it can be shown that the conformal structure on $\partial_{\infty} \mathrm{AdS}_{3}$ is asymptotic in the following sense: if $p_{n}$ is a sequence in $\mathrm{AdS}_{3}$ converging to $p \in \partial_{\infty} \mathrm{AdS}_{3}$ and $v_{n} \in T_{p_{n}} \mathrm{AdS}_{3}$ is a sequence of time-like vectors converging to $v \in T_{p}\left(\partial_{\infty} \mathrm{AdS}_{3}\right)$, then $v$ is not space-like.

\section{Earthquakes and Bent Surfaces in $\mathrm{AdS}_{3}$}

\subsection{Bent surfaces in $\mathrm{AdS}_{3}$}

An embedded topological surface $S \subset \mathrm{AdS}_{3}$ is achronal if geodesics joining two points of $S$ are not time-like. This definition is different from the standard definition of achronality in Lorentzian geometry - in fact achronality does not makes sense in $\mathrm{AdS}_{3}$ since the future of every point is the whole $\mathrm{AdS}_{3}$. On the other hand, if $S$ is achronal in this sense, then it is achronal in the standard sense in some neighbourhood.

If $S$ is achronal, then every small neighborhood $U$ of any point $p \in S$ is disconnected by $S$ in two components: one is the future of $S$ in $U$ and the other is the past of $S$ in $U$. We say that $S$ is past convex (resp. future convex) if there is a family of neighborhoods $\left\{U_{i}\right\}$ that covers $S$ and such that for every $p, q \in S \cap U_{i}$ the geodesic segment joining $p$ to $q$ does not contain points in the future (resp. in the past) of $S$ in $U_{i}$.

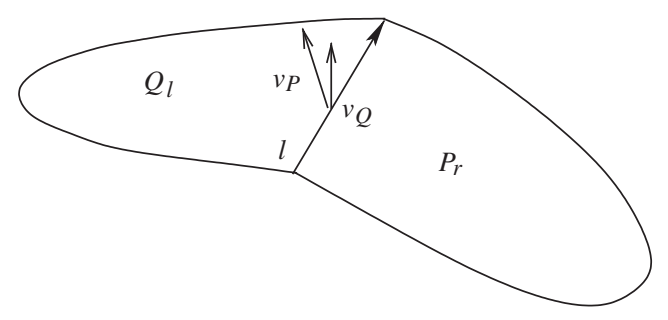

The surface $S$ is past convex iff for every point $p \in S$ there is a space-like plane $P$ such that $P \cap S$ is a convex set of $P$, and planes obtained by moving $P$ slightly in the future do not meet $U_{i} \cap S$. We say that $P$ is a support plane for $S$ in $p$. Notice that in general there are several support planes passing through a point $p \in S$. 
Remark 5.1. Let $l=P \cap Q$ be oriented so that $\alpha(P, Q)>0$. Consider the component, of $P \backslash l$, say $P_{r}$, on the right side of $l$ and the component of $Q \backslash l$, say $Q_{l}$, on the left side of $l$. Then the surface

$$
S=P_{r} \cup l \cup Q_{l}
$$

is past convex. In fact, we need to check the convexity only around points on $l$. But if we slightly move $P$ in the future then the intersection with both $P_{r}$ and $Q_{l}$ is empty.

A past bent surface (resp. future bent surface) in $\mathrm{AdS}_{3}$ is a topological embedding

$$
b: \mathcal{H} \rightarrow \mathrm{AdS}_{3}
$$

where $\mathcal{H}$ is an open convex subset of $\mathbb{H}^{2}$ with geodesic boundary and $b$ satisfies the following conditions:

- There is a geodesic lamination $L$ of $\mathcal{H}$ such that the restriction of $b$ on each connected component of $\mathcal{H} \backslash L$ is isometric and totally geodesic.

- Each leaf of $L$ is isometrically sent to a geodesic of $\mathrm{AdS}_{3}$.

- The image of $b$ is past convex (resp. future convex).

Remark 5.2. A natural question is whether the map $b$ extends to the boundary. If a boundary component $l$ of $\mathcal{H}$ is a boundary component of some stratum of $L$, then it is clear that $b$ extends on $l$.

Instead, if there is a sequence of leaves $l_{n} \in L$ converging to $l$, then there are several possibilities:

(1) $b\left(l_{n}\right)$ converges to a space-like geodesic in $\mathrm{AdS}_{3}$;

(2) $b\left(l_{n}\right)$ converges to a point in $\partial_{\infty} \mathrm{AdS}_{3}$;

(3) $b\left(l_{n}\right)$ converges to a light-like segment in $\partial_{\infty} \mathrm{AdS}_{3}$.

It is then clear that not in all cases the map $b$ can be extended on the boundary.

There is a transverse measure on $L$ that encodes the amount of bending along $L$. When $L$ is locally finite, there is a fairly simple way to describe this measure. Given a leaf $l$, there are exactly two regions $F, F^{\prime}$ bounded by $l$, then the weight of $l$ is simply the 
bending angle between the space-like planes containing $b(F)$ and $b\left(F^{\prime}\right)$. In the general case the measure is defined by an approximation argument using the fact that if $P, O, R$ are space-like planes such that the intersection $P \cap Q$ lies above $R$, then the bending angle between $P$ and $Q$ is greater than the sum of bending angles between $P$ and $R$ and between $R$ and $Q$ (see [5] to check details).

If $c:[0,1] \rightarrow \mathcal{H}$ is a path transverse to $L$, then for every partition $I=\left(t_{0}=0<\right.$ $\left.t_{1}<\ldots<t_{k}=1\right)$ one defines $\mu(c ; I)$ as the sum of the bending angles between support planes at $b\left(c\left(t_{i}\right)\right)$ and $b\left(c\left(t_{i+1}\right)\right.$. If $I^{\prime}$ is finer than $I$, the property expressed above shows that $\mu\left(c, I^{\prime}\right) \leq \mu(c, I)$, so the mass of $c$ is defined as

$$
\mu(C)=\inf _{I} \mu(C ; I)=\lim _{|I| \rightarrow 0} \mu(C ; I) .
$$

\subsection{From earthquakes to bent surfaces}

Given two metrics $\eta_{l}$ and $\eta_{r}$ in $\mathcal{T}_{g, n}$, let $h_{1}, h_{\mathrm{r}}: \pi_{1}(\Sigma) \rightarrow P S L_{2}(\mathbb{R})$ be the corresponding holonomies. We consider the isometric action of $\pi_{1}(\Sigma)$ on $\operatorname{AdS}_{3}$ given by the product holonomy

$$
\left(h_{1}, h_{\mathrm{r}}\right): \pi_{1}(\Sigma) \rightarrow P S L_{2}(\mathbb{R}) \times P S L_{2}(\mathbb{R})
$$

In this section, we will associate to every right earthquake transforming $\Sigma_{\eta l}$ into $\Sigma_{\eta_{r}}$ a past bent surface that is invariant under the representation $\left(h_{\mathrm{l}}, h_{\mathrm{r}}\right)$ and we will show that this bent surface is sufficient to recover the earthquake.

Take a measured geodesic lamination $\lambda$ on $\Sigma_{\eta_{l}}$ such that the right earthquake along $\lambda$ transforms $\Sigma_{\eta_{l}}$ to $\Sigma_{\eta_{r}}$ :

$$
E_{\lambda}^{r}: \Sigma_{\eta_{l}} \rightarrow \Sigma_{\eta_{r}}
$$

The lifting of $E_{\lambda}^{r}$ to the universal covering is a map

$$
\tilde{E}: \mathcal{H}_{l} \rightarrow \mathbb{H}^{2}
$$

that satisfies the following properties, already mentioned in the previous section.

- The image of $\tilde{E}$ is the universal cover $\mathcal{H}_{r}$ of $\Sigma_{\eta_{r}}$.

- $\tilde{E} \circ h_{\mathrm{l}}(\gamma)=h_{\mathrm{r}}(\gamma) \circ \tilde{E}$.

- The image of $\tilde{E}$ is the convex hull of the limit set of $h_{\mathrm{r}}$. 
- For every component $F$ of $\mathbb{H}^{2} \backslash \tilde{\lambda}$, there is an element $A=A(F)$ in $P S L_{2}(\mathbb{R})$ such that $\left.\tilde{E}\right|_{F}=\left.A\right|_{F}$.

- $A\left(h_{\mathrm{l}}(\gamma)(F)\right)=h_{\mathrm{r}}(\gamma) \circ A(F)$.

- If $F, F^{\prime}$ are two components of $\mathcal{H} \backslash \tilde{\lambda}$, then the comparison isometry $B^{*}=$ $A(F)^{-1} \circ A\left(F^{\prime}\right)$ is a hyperbolic transformation whose axis separates $F$ from $F^{\prime}$. If the axis $l$ of $B^{*}$ is oriented from the repulsive fixed point towards the attractive fixed point, then $F^{\prime}$ is on the left side of $l$ whereas $F$ is on the right side.

- If $F$ and $F^{\prime}$ are adjacent, then the axis of $B^{*}$ is the common edge $e$ and the translation length of $B^{*}$ is the weight of $e$.

Given any component $F$ in $\mathcal{H}_{l} \backslash \tilde{\lambda}$, let us take the set of its ideal vertices $\left\{x_{i}\right\} \subset \partial \mathbb{H}^{2}$. Setting $A=A(F)$, we can consider on the plane $P_{A}$ the convex hull of the set $\left\{\left(x_{i}, A x_{i}\right)\right\}$, that is a space-like geodesic polygon in $\mathrm{AdS}_{3}$, say $K(F)$.

Let $S$ be the closure of the union of all $K(F)$ 's.

Proposition 5.3. $S$ is a future convex bent surface in $\mathrm{AdS}_{3}$ that is invariant under the action of $\pi_{1}(S)$.

Moreover, if $\mathcal{H}$ denotes the universal covering of $E_{\lambda / 2}^{r}\left(\Sigma_{\eta_{l}}\right)$ then there is a bending map

$$
\iota: \mathcal{H} \rightarrow S
$$

that is equivariant under the $\pi_{1}(\Sigma)$ action.

The bending lamination associated to $\iota$ is the image throughout the earthquake map $E: \mathcal{H}_{l} \rightarrow \mathcal{H}$ of the lamination $\tilde{\lambda} / 2$.

To prove Proposition 5.3, we need the following elementary facts of AdS geometry, the proofs can be found in [5].

Lemma 5.4. Let $l$ be a complete geodesic line in $\mathrm{AdS}_{3}$ with endpoints $p=(x, y)$ and $q=\left(x^{\prime}, y^{\prime}\right)$. Let $s_{l}, s_{r}$ be, respectively, the geodesics of $\mathbb{H}^{2}$ with endpoints $x, x^{\prime}$ and $y, y^{\prime}$.

The connected component of the stabilizer of $l$ in $P S L_{2}(\mathbb{R}) \times P S L_{2}(\mathbb{R})$ is the set of pairs $(A, B)$ where $A$ is a hyperbolic transformation with axis $s_{l}$ and $B$ is a hyperbolic transformation with axis $s_{r}$. 
Let us orient $l, s_{l}$, and $s_{r}$ in such a way that the corresponding starting points are, respectively, $(x, y), x$, and $y$. Given a transformation $A$ (resp. $B$ ) with axis $s_{l}$ (resp. $s_{r}$ ), let $t(A) \in \mathbb{R}$ (resp. $\left.t(B)\right)$ denote the signed translation length $(t(A)$ is positive if $x$ is the repulsive fixed point, negative otherwise). Then the transformation $(A, B)$ acts as a translation on $l$ of factor $(t(A)+t(B)) / 2$. The rotation angle of $(A, B)$ along $l$ is $(t(B)-t(A)) / 2$.

Definition 5.5. If $(A, B)$ preserves $l$, the rotation angle of $(A, B)$ along $l$ is the signed bending angle formed by a space-like plane $P$ containing $l$ with its image $(A, B) \cdot P$.

We prove now Proposition 5.3.

Proof of Proposition 5.3. We prove the statement assuming that $\lambda$ is locally finite. The general case will follow by an approximation argument.

For each face of $\mathcal{H}_{l} \backslash \tilde{\lambda}$ let $r_{F}: \mathbb{H}^{2} \rightarrow \mathrm{AdS}_{3}$ be the isometric embedding whose trace at infinity is the map

$$
X \mapsto(X, A(F) X)
$$

Clearly, we have that $K(F)=r_{F}(F)$.

Given two strata $F, F^{\prime}$ we have that $r_{F^{\prime}}=(1, B) \circ r_{F}$ where $B=A\left(F^{\prime}\right) \circ A(F)^{-1}$. Thus, $K\left(F^{\prime}\right)$ is obtained by applying the transformation $(1, B)$ to $r_{F}\left(F^{\prime}\right)$.

Notice that $B=A(F) B^{*} A(F)^{-1}$ where $B^{*}=A(F)^{-1} A\left(F^{\prime}\right)$ is the comparison isometry.

Let $l$ be the image through $r_{F}$ of the axis of $B^{*}$, that is the geodesic with endpoints $p_{-}=\left(X_{-}\left(B^{*}\right), A(F) X_{-}\left(B^{*}\right)\right)=\left(x_{-}\left(B^{*}\right), X_{-}(B)\right)$ and $p_{+}=\left(x_{+}\left(B^{*}\right), A(F) X_{+}\left(B^{*}\right)\right)=$ $\left(X_{+}\left(B^{*}\right), x_{+}(B)\right)$. Notice that both $p_{-}$and $p_{+}$are fixed by $(1, B)$. Thus, $l$ is left invariant by $(1, B)$.

Now if $P_{l}$ and $P_{r}$ denote the half-planes bounded by $l$ on $P_{A(F)}$, then $K(F)=r_{F}(F)$ is contained in $P_{r}$ whereas $r_{F}\left(F^{\prime}\right)$ is contained in $P_{l}$.

We can conclude that

$$
K(F) \cup K\left(F^{\prime}\right) \subset P_{r} \cup(1, B) P_{l} .
$$

This shows that if $F$ and $F^{\prime}$ are not adjacent, then $K(F)$ and $K\left(F^{\prime}\right)$ are disjoint. When $F$ and $F^{\prime}$ meet along a line, this line is the axis of $B$, so $K(F)$ and $K\left(F^{\prime}\right)$ meet along the line 
$l$. In this case by Lemma 5.4, the bending angles formed along $l$ between $K(F)$ and $K\left(F^{\prime}\right)$ is equal to $t(B) / 2=t\left(B^{*}\right) / 2$, that is to one-half the mass of the line $F \cap F^{\prime}$.

Since $P_{r} \cup(1, B) P_{l}$ is achronal, it turns out that $S$ is achronal.

Notice that we have that

$$
K\left(h_{\mathrm{l}}(\gamma)(F)\right)=\left(h_{\mathrm{l}}(\gamma), h_{\mathrm{r}}(\gamma)\right) K(F)
$$

thus $S$ is invariant.

In order to show that $S$ is a past bent surface, we need to construct the bending map.

We could try to glue the maps $r_{F}$. However if $F$ and $F^{\prime}$ are adjacent, for $p$ in $F \cap F^{\prime}$ we have

$$
r_{F^{\prime}}(p)=(1, B) r_{F}(p)
$$

Notice that both $r_{F}(p)$ and $r_{F^{\prime}}(p)$ are contained in the geodesic $l$ described above. On the other hand by Lemma 5.4, the transformation $(1, B)$ acts by a translation of factor $t(B) / 2$ on $l$. Thus, the maps $r_{F}$ do not glue to an isometric map from $\mathcal{H}_{l}$ into $\mathrm{AdS}_{3}$.

On the other hand, these maps can be glued if each component of $\mathcal{H}_{l} \backslash \tilde{\lambda}$ is identified to the adjacent components through a right translation of length equal to the mass of the corresponding edge divided by 2 . This shows that the maps $r_{F}$ induce a continuous isometric identification

$$
\mathcal{H} \rightarrow S
$$

To conclude, we have to prove that $S$ is past convex. It is sufficient to show that $S$ is convex at each point in $K(F) \cap K\left(F^{\prime}\right)$ where $F$ and $F^{\prime}$ are two adjacent components of $\mathcal{H}_{l} \backslash \lambda$. On the other hand by (2), we have that $K(F) \cup K\left(F^{\prime}\right)$ is contained in $P_{r} \cup(1, B) P_{l}$. By Lemma 5.4, the angle formed between $P_{A(F)}$ and $(1, B) P_{A(F)}$ is positive (with respect to the natural orientation of $l$ ). Thus, Remark 5.1 shows that $P_{r} \cup(1, B) P_{l}$ is past convex and this concludes the proof.

Remark 5.6. The surface $S$ is well defined up to post-composition with an isometry of $\mathrm{AdS}_{3}$. Notice that different earthquakes produce different bent surfaces. This depends on the fact that the shearing lamination of the earthquake $E^{r}: \Sigma_{\eta_{l}} \rightarrow \Sigma_{\eta_{r}}$ is explicitly related to the bending lamination of $S$. 
It could be proved that every bent convex surface can be associated to an earthquake. More precisely, the following statement holds.

Proposition 5.7. Consider an equivariant bent surface

$$
b: \mathcal{H} \rightarrow \mathrm{AdS}_{3}
$$

such that $S_{+}=\mathcal{H} /\left(h_{1}, h_{\mathrm{r}}\right)$ is an admissible surface. Let $\lambda_{+}$be the lamination on $S$ corresponding to the bending lamination on $H$. Then

$$
E_{\lambda}^{r}\left(S_{+}\right)=S_{l}, \quad E_{\lambda}^{l}\left(S_{+}\right)=S_{r}
$$

Since this proposition is not strictly necessary for the proof of Theorem 1.2, we skip the proof referring to [5].

\section{Bent Surfaces in $\mathrm{AdS}_{3}$ and Achronal Meridians in $\partial_{\infty} \mathrm{AdS}_{3}$}

This section analyzes the relationship between bent surfaces in the AdS space and curves of a certain type-called achronal meridians-arising as their boundary at infinity.

\subsection{Achronal meridians as graph of cyclic-order preserving maps of $\partial \mathbb{H}^{2}$}

In this section, it will be convenient to fix a universal covering of $\partial \mathbb{H}^{2} \times \partial \mathbb{H}^{2}$. In particular, we fix a point $q_{0}$ in $\mathbb{H}^{2}$ and we consider the visual angle on $\partial \mathbb{H}^{2}$ with respect to $q_{0}$. This gives a natural covering map $p: \mathbb{R} \rightarrow \partial \mathbb{H}^{2}$. Clearly, we can consider the product covering $\mathbb{R}^{2} \rightarrow \partial \mathbb{H}^{2} \times \partial \mathbb{H}^{2}$ sending $(\theta, \phi)$ to $(p(\theta), p(\phi))$. For notation convenience, we slightly modify this covering by considering the map

$$
\mathbb{R}^{2} \ni(x, y) \mapsto(p(2 \pi x), p(2 \pi y)) \in \partial \mathbb{H}^{2} \times \partial \mathbb{H}^{2} .
$$

In this way, the covering transformations are translations with integer coordinates.

By definition, space-like curves on $\partial_{\infty} \mathrm{AdS}_{3}$ correspond to curves $(x(t), y(t))$ such that $x^{\prime}(t) y^{\prime}(t)>0$. They are locally graphs of orientation-preserving maps between two open intervals of $\partial \mathbb{H}^{2}$. Thus, the lifting on the universal covering $\mathbb{R}^{2}$ of a space-like curve $c$ is the graph of a strictly increasing function

$$
f: \mathbb{R} \rightarrow \mathbb{R}
$$

such that $f(x+n)=f(x)+m$ for some $n, m \in \mathbb{Z}$ depending on the homology class of $c$. 
Space-like meridians in $\partial_{\infty} \mathrm{AdS}_{3}$ are graphs of orientation-preserving diffeomorphisms of $\partial \mathbb{H}^{2}$, since their liftings correspond to graphs of orientation-preserving diffeomorphisms $f: \mathbb{R} \rightarrow \mathbb{R}$ such that $f(x+1)=f(x)+1$.

Limit of space-like meridians are locally achronal meridian. A meridian is locally achronal if for every $p \in c$ there is a neighborhood $U \subset \partial_{\infty} \mathrm{AdS}_{3}$ such that no pair of points $q, r \in C \cap U$ is related by a time-like $\operatorname{arc}$ in $U$. It can be shown that locally achronal meridians correspond to monotonically increasing (possibly discontinuous) functions

$$
f: \mathbb{R} \rightarrow \mathbb{R}
$$

such that $f(x+1)=f(x)+1$. Indeed given such a function, we can consider the subset in $\mathbb{R}^{2}$

$$
G_{f}=\left\{(x, y) \mid \lim _{t \rightarrow X_{-}} f(t) \leq y \leq \lim _{t \rightarrow X_{+}} f(t)\right\} .
$$

Lemma 6.1. $G_{f}$ is a connected embedded curve in $\mathbb{R}^{2}$.

Proof. If $f_{n}$ is a sequence of continuous monotonically increasing functions approximating $f$ pointwise, the length of the graph of $f_{n}$ on some interval $[a, b]$ is bounded by $(b-a)+\left(f_{n}(a)-f_{n}(b)\right)$ so it is uniformly bounded. Since graph $\left(\left.f_{n}\right|_{[a, b]}\right)$ stays in some compact set of $\mathbb{R}^{2}$ it converges to a topological curve. Such a curve coincides with $\left.G_{f}\right|_{[a, b]}$.

The fact that $G_{f}$ is embedded is due to the fact that every point of $G_{f}$ disconnects $G_{f}$ in exactly two components.

Given an increasing function $f$, the set $G_{f}$ projects to an embedded closed curve in $\partial \mathbb{H}^{2}$ provided that $f$ is not constant on some interval of length bigger than 1 or discontinuity points with jumps bigger than 1 . On the other hand by our assumption $f(x+1)=f(x)+1$, it is easy to see that this can happen if and only if up to some translation we have $f(t)=[t+c]+c^{\prime}$ for some constants $c, c^{\prime}$. In both these cases, $G_{f}$ projects in the union of two leaves in $\partial \mathbb{H}^{2}$.

In all the other cases, $G_{f}$ projects to a locally achronal meridian in $\partial \mathbb{H}^{2} \times \partial \mathbb{H}^{2}$. Conversely, every locally achronal curve arises in this way. Namely, given an achronal meridian $C$ we define $f: \mathbb{R} \rightarrow \mathbb{R}$ by setting $f(x)=\sup \{y \mid(x, y) \in \tilde{C}\}$ where $\tilde{C}$ is a component of the pre-image of $C$ in $\mathbb{R}^{2}$. Since $C$ meets every leaf, the map is well defined. 
Since $C$ is a meridian, $f(x+1)=f(x)+1$. Finally, since $C$ is achronal $f$ turns out to be increasing and $C$ coincides with the projection of $G_{f}$.

With some abuse, we call $G_{f}$ the graph of the function $f$ (notice that $G_{f}$ coincides with the standard graph when $f$ is continuous).

We collect some facts about locally achronal meridians that will be useful in what follows.

Lemma 6.2. If $C$ is an achronal meridian, there exists a projective plane $P$ whose intersection with $\mathrm{AdS}_{3}$ is space-like and such that $C \cap P=\emptyset$.

Proof. Let $f: \mathbb{R} \rightarrow \mathbb{R}$ be the increasing function such that $C$ is the projection of $G_{f}$. Acting by isometries on $C$, we can suppose that $f$ is continuous at 0 and $1 / 2$ and $f(0)=0$ (so $f(1)=1$ ) and $f(1 / 2)=1 / 2$. Notice that $G_{f} \cap[0,1]^{2}$ is contained in the two squares $Q_{1}=[0,1 / 2] \times[0,1 / 2]$ and $Q_{2}=[1 / 2,1] \times[1 / 2,1]$. Moreover, by our assumption on the continuity points $(1 / 2,0),(0,1 / 2),(1,1 / 2)$, and $(1 / 2,1)$ do not lie in $G_{f}$.

Thus, the line of equation $y=x+1 / 2$ is disjoint from $G_{f}$. The projection $l$ of such a line on $\partial \mathbb{H}^{2} \times \partial \mathbb{H}^{2}$ is the graph of the trace at infinity of the rotation of angle $\pi$ about the point $q_{0}$. Thus, there is a space-like plane $P$ such that $l=P \cap \partial_{\infty} \mathrm{AdS}_{3}$. It follows that $P \cap C=\emptyset$.

Since $C$ does not intersect $P$, we can consider the convex hull $K$ of $C$ in the affine chart $\mathbb{R}^{3}=\mathbb{R} P^{3} \backslash P$. It is easy to see that $K$ does not depend on the plane $P$ (this because the change of chart map between $\mathbb{R}^{3} \backslash P$ and $\mathbb{R}^{3} \backslash Q$ sends compact convex sets disjoint from $Q$ into compact convex sets).

Lemma 6.3. $K$ is contained in $\overline{\mathrm{AdS}_{3}}$. More precisely

(1) the interior of $K$ is contained in $\mathrm{AdS}_{3}$

(2) the intersection of the boundary of $K$ with $\partial_{\infty} \mathrm{AdS}_{3}$ is $C$.

Proof. Given a point $p=(x, y)$ in $\partial \mathrm{AdS}_{3} \backslash C$, we claim that it does not lie in $K$. As a consequence, we have that $K \cap \partial_{\infty} \mathrm{AdS}_{3}=C$. In particular, $K$ is contained in the closure of one component of $\mathbb{R}^{3} \backslash \partial_{\infty} \mathrm{AdS}_{3}$. Since the curve $C$ is not trivial in $\mathbb{R}^{3} \backslash \mathrm{AdS}_{3}, K$ must be contained in $\mathrm{AdS}_{3}$.

We now prove the claim. Let us consider a time-like plane, say $Q$, through $p$. For homological reasons $Q$ must intersect $C$ in two points $q=(u, v), q^{\prime}=\left(u^{\prime}, v^{\prime}\right)$. 
Since $Q \cap \partial_{\infty} \mathrm{AdS}_{3}$ is the graph of an orientation-reversing diffeomorphism of $\partial \mathbb{H}^{2}$, we can fix $q, q^{\prime}$ so that $x$ is contained in the positive segment $\left[u, u^{\prime}\right]$ whereas $y$ is in the positive segment $\left[v^{\prime}, v\right]$.

Up to the action of $P S L_{2}(\mathbb{R}) \times P S L_{2}(\mathbb{R})$, we can also suppose that the points

$$
\hat{q}=(0,0), \quad \hat{p}=(1 / 3,2 / 3), \quad \hat{q}^{\prime}=(2 / 3,1 / 3)
$$

project respectively to $q, p, q^{\prime}$. Let $\tilde{C}$ be the lifting of $C$ passing through $(0,0)$. We have that $\tilde{C} \cap[0,1]^{2}$ is contained in $R=[0,2 / 3] \times[0,1 / 3] \cup[2 / 3,1] \times[1 / 3,1]$. Consider the line $\hat{l}$ of equation $y=x+1 / 3$. There is a space-like plane $P$ such that $P \cap \partial_{\infty} \mathrm{AdS}_{3}$ is the projection of $\hat{l}$. If the points $(0,1 / 3),(2 / 3,0),(2 / 3,1)$ do not lie on $\tilde{C}$, the plane $P$ is disjoint from $C$, so it is disjoint form $K$. Otherwise, since $\hat{l}$ does not disconnect $\tilde{C}$, the plane $P$ is a support plane for $K$. Notice that in this case $K \cap P$ is the convex hull of $C \cap P$. Since $\partial_{\infty} \mathrm{AdS}_{3} \cap P$ is strictly convex, we have that $p$ does not lie on $K \cap P$, so $p \notin K$.

Remark 6.4. Support planes of $K$ cannot be time-like, indeed for homological reasons the transverse intersection of $C$ with a time-like plane is not empty.

$K$ is a plane if and only if $C$ lies in some projective plane, otherwise it is a closed ball (in $\mathbb{R} P^{3}$ ). The boundary of $K$ in $\mathrm{AdS}_{3}$ has two connected components. By the remark above, both components are achronal surfaces. More precisely, one component is past convex and the other is future convex.

The upper boundary of $K$-denoted by $\partial_{+} K$-is the past convex component of $\partial K$. Analogously, the lower boundary of $K$-denoted by $\partial_{-} K$-is the future convex component of $\partial K$.

We say that a support plane of $K$ is an upper (resp. lower) support plane if it is a support for the upper boundary (resp. lower boundary).

Remark 6.5. Let $f: \mathbb{R} \rightarrow \mathbb{R}$ be an increasing function such that $C$ is the projection of $G_{f}$. Given $A \in P S L_{2}(\mathbb{R})$, the following conditions are equivalent:

(1) $P_{A}$ is a upper (resp. lower) support plane for $C$.

(2) There is a lifting of $A$ to $\mathbb{R}$ such that $f_{A}=\tilde{A}^{-1} \circ f$ satisfies $f_{A}(x) \leq x$ (resp. $\left.f_{A}(x) \geq x\right)$ and admits two fixed points on $[0,1)$.

If $P$ is a support plane for $K$, the intersection $P \cap K$ is the convex hull of $P \cap C$. 
If $P$ is a space-like support plane the intersection of $P$ with $\partial K$ is either a geodesic line or a hyperbolic ideal polygon.

If $P$ is a light-like support plane, it is tangent to $\partial_{\infty} \mathrm{AdS}_{3}$ at some point $p$ in $C$. Moreover, since $P$ meets the boundary of $K$, there are points $q, r$ of $C$ lying respectively on the left and the right leaf through $p$. The intersection of $P$ with $C$ is a light-like triangle with two ideal edges (that means that two edges are segments of leaves of the double foliation of $\partial_{\infty} \mathrm{AdS}_{3}$ ).

It turns out that each boundary component of $K$ is the union of a space-like region formed by the set of points admitting only space-like support planes and some ideal light-like triangles.

The space-like part is a union of space-like geodesics and of ideal hyperbolic polygons. The boundary of the space-like part in $\partial_{+} K$ is the union of the space-like edges of the ideal light-like triangles contained in $\partial_{+} K$.

In what follows, we will need the following technical fact.

Lemma 6.6. Let $C$ be an achronal meridian and let $K$ denote its convex hull. If $P$ and $Q$ are space-like upper support planes, then they intersect along a line $l$. Moreover, if $l$ is oriented so that $\alpha(P, Q)>0$ then $P \cap K$ is contained in the right side of $l$ in $P$ and $Q \cap K$ is contained in the left side of $l$ in $Q$.

Proof. Suppose that two upper support planes $P, Q$ are disjoint in $\mathrm{AdS}_{3}$. Then there are planes $P^{\prime}, Q^{\prime}$ obtained by slightly moving in the future of $P$ and $Q$, respectively, such that $P^{\prime} \cap K=Q^{\prime} \cap K=\emptyset$ and we can moreover suppose that $P^{\prime} \cap Q^{\prime}=P^{\prime} \cap Q=Q^{\prime} \cap P=\emptyset$. Notice that $\mathrm{AdS}_{3} \backslash\left(P^{\prime} \cup Q^{\prime}\right)$ is the disjoint union of two cylinders and $P$ and $Q$ lie in different components. Now $K$ is a connected set in $\operatorname{AdS}_{3} \backslash\left(P^{\prime} \cup Q^{\prime}\right)$ that contains a point on $P$ and a point on $Q$, so it intersects the two components of $\operatorname{AdS}_{3} \backslash\left(P^{\prime} \cup Q^{\prime}\right)$ and this gives a contradiction.

To conclude the proof of the lemma, it is sufficient to notice that given $p \in P$ and $q \in Q$, the only possibility that the geodesic segment $[p, q]$ intersects neither $P^{\prime}$ nor $Q^{\prime}$ is that $p \in P_{r}$ and $q \in Q_{l}$.

Remark 6.7. Let $P, Q$, and $l$ be as in Lemma 6.6. Let $p_{-}=\left(x_{-}, y_{-}\right)$and $p_{+}=\left(x_{+}, y_{+}\right)$be, respectively, the starting and the ending points of $l$. Let $s$ be the geodesic in $\mathbb{H}^{2}$ with endpoints $x_{-}$and $x_{+}$(oriented from $x_{-}$to $x_{+}$). Then if $P \cap C=\left\{\left(x_{i}, y_{i}\right)\right\}_{i \in I}$ and $Q \cap C=$ $\left.\left\{x_{j}^{\prime}, y_{j}^{\prime}\right)\right\}_{j \in J}$, points $x_{i}$ lie on the right side of $s$ whereas points $x_{j}^{\prime}$ lie on the left side of $s$. 
In particular, $s$ weakly separates the convex hull of points $x_{i}$ from the convex hull of points $x_{j}^{\prime}$.

\subsection{From bent surfaces to achronal meridians}

Let

$$
E^{r}: \Sigma_{\eta_{l}} \rightarrow \Sigma_{\eta_{r}}
$$

be a right earthquake between two admissible surfaces. In this section, an achronal curve $C$ will be associated to $E^{r}$ and we will show that such a curve determines the earthquake.

More precisely, we consider the equivariant bent surface

$$
b: \mathcal{H} \rightarrow \mathrm{AdS}_{3}
$$

associated to $E^{r}$ in Section 5.2. We will construct an achronal meridian $C$ such that $b(\mathcal{H})$ turns out to be the space-like region of the convex hull of $C$.

First, we consider the trace at infinity of the earthquake. Contrary to the "classical" case (where the source and the target spaces are the whole $\mathbb{H}^{2}$ ), it is not true that the map $E^{r}$ extends by continuity on the closure of $\mathcal{H}$ at infinity. Nevertheless the map $E^{r}$ extends on the set of ideal points of every stratum of the fault lamination. So we consider the following set in $\partial_{\infty} \mathrm{AdS}_{3}$

$$
\partial_{\infty} \mathcal{H}=\left\{\left(x, E^{r}(x)\right) \mid x \in F \cap \partial \mathbb{H}^{2}, F \text { is a stratum of the fault lamination }\right\}
$$

This notation is due to the fact that $\partial_{\infty} \mathcal{H}$ can be regarded as the set of ideal points of the bent surface $b(\mathcal{H})$.

More precisely, for every stratum of the fault lamination $F$, we consider the set $\partial_{\infty} K(F)$ of ideal points of $K(F)$ where $K(F)$ is the face of $b(\mathcal{H})$ corresponding to $F$. It turns out that

$$
\partial_{\infty} \mathcal{H}=\bigcup \partial_{\infty} K(F)
$$

Remark 6.8. If $h_{1}$ and $h_{\mathrm{r}}$ are the holonomies of $\eta_{l}$ and $\eta_{r}$, then $\partial_{\infty} \mathcal{H}$ is invariant for the representation $\left(h_{1}, h_{\mathrm{r}}\right)$. 


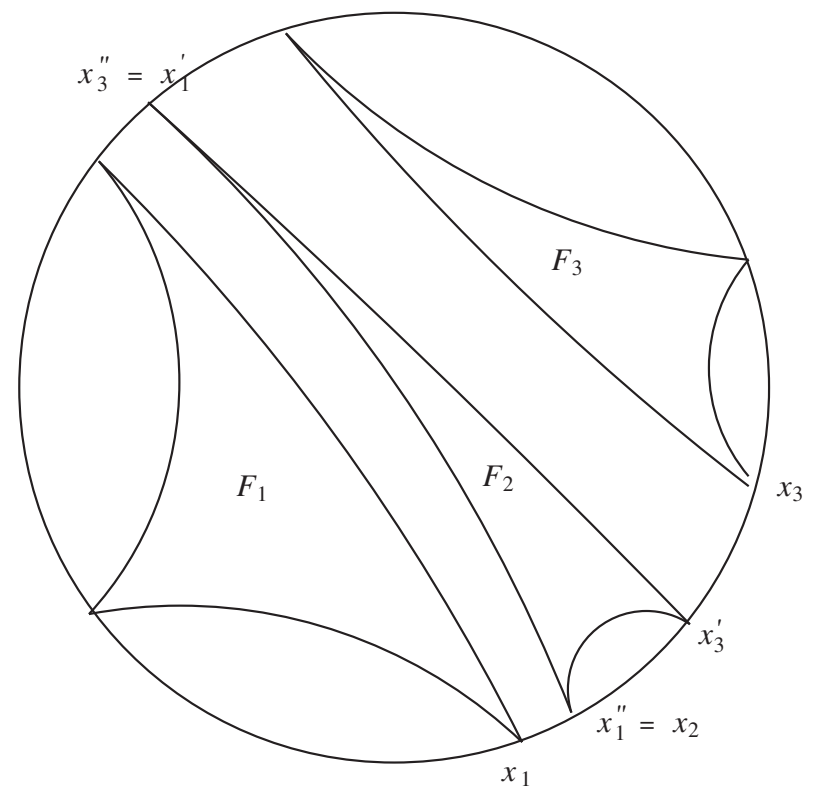

Given three points $x, y, z \in \partial \mathbb{H}^{2}$ such that $x \neq z$, we write $x \leq y \leq z$ if $y$ lies in the positive closed segment in $\partial \mathbb{H}^{2}$ with first endpoint $x$ and second endpoint $z$. We write $x<y<z$ if $x \leq y \leq z$ and $y \neq x$ and $y \neq z$. The set $\partial_{\infty} \mathcal{H}$ satisfies the following property:

Lemma 6.9. Given three points $p_{1}, p_{2}, p_{3} \in \partial_{\infty} \mathcal{H}$ such that $p_{i}=\left(x_{i}, y_{i}\right) \in \partial_{\infty} \mathcal{H}$ and $x_{1}<$ $x_{2}<x_{3}$ then $y_{1}<y_{2}<y_{3}$.

Proof. Let $F_{i}$ be the stratum of the fault lamination such that $p_{i} \in K\left(F_{i}\right)$. We prove the statement assuming $F_{1} \neq F_{2} \neq F_{3}$. The other cases are simpler and quite similar.

Up to applying some cyclic permutation of indices, we may suppose that $F_{2}$ separates $F_{1}$ from $F_{3}$. This precisely means that there are points in $\partial_{\infty} F_{2}$, say $x_{1}^{\prime}, x_{1}^{\prime \prime}, x_{3}^{\prime}, x_{3}^{\prime \prime}$ such that

$$
x_{1}^{\prime} \leq x_{1} \leq x_{1}^{\prime \prime}, \quad x_{3}^{\prime} \leq x_{3} \leq x_{3}^{\prime \prime}
$$

and the positive intervals $\left(x_{1}^{\prime}, x_{1}^{\prime \prime}\right)$ and $\left(x_{3}^{\prime}, x_{3}^{\prime \prime}\right)$ are disjoint and do not contain points in $\partial_{\infty} F_{2}$. Moreover by the hypothesis, either $x_{1}^{\prime \prime}=x_{2}=x_{3}^{\prime}$ or $x_{1}^{\prime \prime} \leq x_{2} \leq x_{3}^{\prime}$. 
Now, let $A \in P S L_{2}(\mathbb{R})$ such that $\left.E^{r}\right|_{F_{2}}=A$. By properties of the earthquake $A^{-1} E^{r}\left(x_{i}\right)$ is contained in $\left[x_{i}^{\prime}, x_{i}^{\prime \prime}\right]$ for $i=1,3$, therefore we have

$$
A^{-1} y_{1}<X_{2}<A^{-1} y_{3} .
$$

Since $A$ is an orientation-preserving diffeomorphism of $\partial \mathbb{H}^{2}$, we conclude that

$$
y_{1}<y_{2}<y_{3} .
$$

The property expressed in Lemma 6.9 is shared in some weaker form by all subsets of any achronal meridian.

Lemma 6.10. If $C$ is an achronal meridian in $\partial_{\infty} \mathrm{AdS}_{3}$, then given three points $p_{1}, p_{2}, p_{3} \in C$ such that $p_{i}=\left(x_{i}, y_{i}\right)$ with $x_{1}<x_{2}<x_{3}$ then either $y_{1}=y_{2}=y_{3}$ or $y_{1} \leq y_{2} \leq$ $y_{3}$.

Proof. It is sufficient to notice that $C$ is the projection of some curve $G_{f}$ where $f: \mathbb{R} \rightarrow$ $\mathbb{R}$ is an increasing function such that $f(x+1)=f(x)+1$.

Definition 6.11. A subset of $\partial_{\infty} \mathrm{AdS}_{3}$ that is not contained in the union of any left and right leaves and satisfies the conclusion of Lemma 6.10 will be said to be connectible by an achronal meridian.

Lemma 6.12. If $\Delta \subset \partial_{\infty} \mathrm{AdS}_{3}$ is connectible by an achronal meridian, then there is an achronal meridian passing through every point of $\Delta$.

Indeed, there are two extremal possible choices $C_{-}(\Delta), C_{+}(\Delta)$ such that every other choice lies in between them.

Proof. We fix the angular coordinates on $\partial \mathbb{H}^{2} \times \partial \mathbb{H}^{2}$ such that $(0,0)$ corresponds to some point in $\Delta$.

Let $\tilde{\Delta}$ be the pre-image of $\Delta$ on the open square $(0,1) \times(0,1)$ through the covering map $\mathbb{R}^{2} \rightarrow \partial \mathbb{H}^{2} \times \partial \mathbb{H}^{2}$ described in Section 6.1. This set has the property that if $(x, y),\left(x^{\prime}, y^{\prime}\right) \in \tilde{\Delta} \cap(0,1)^{2}$ and $x<x^{\prime}$ then $y \leq y^{\prime}$.

Thus, we can define $f_{-}:[0,1] \rightarrow[0,1]$ by setting

$$
f_{-}(0)=0, \quad f_{-}(1)=1, \quad f_{-}(t)=\sup \{y \mid \exists(x, y) \in \tilde{\Delta} \text { s.t. } x \leq t\}
$$


where we use the convention $\sup \emptyset=0$. This function is clearly increasing, and we can extend $f_{-}$to an increasing function on $\mathbb{R}$ such that $f_{-}(t+1)=f_{-}(t)+1$

If $(x, y) \in \tilde{\Delta}$, then $f(x) \geq y$, on the other hand by the property of $\tilde{\Delta}$, we have that

$$
\lim _{t \rightarrow x_{-}} f_{-}(t) \leq Y
$$

thus $(x, y)$ is contained in $G_{f_{-}}$. Finally, notice that if $(t, 0)$ projects to a point of $\Delta$, then there cannot be any point $(x, y) \in \tilde{\Delta}$ such that $x<t$. It follows that $f=0$ on the interval $[0, t)$, and $(0, t) \times\{0\} \in G_{f}$.

$G_{f}$ projects to some curve $C$ in $\partial \mathbb{H}^{2} \times \partial \mathbb{H}^{2}$. Since $C$ contains $\Delta$, it cannot be the union of any left and right leaves. Thus, $C$ is an achronal meridian containing $\Delta$.

We can also define $f_{+}:[0,1] \rightarrow[0,1]$ by putting

$$
f_{+}(0)=0, \quad f_{+}(1)=1, \quad f_{+}(t)=\inf \{y \mid \exists(x, y) \in \tilde{\Delta} \text { s.t. } t \leq x\},
$$

where we use the convention that $\inf \emptyset=1$. The same argument used above shows that this function is increasing and that the corresponding achronal meridian contains $\Delta$.

Let $C$ be an achronal meridian containing $\Delta$. There is a monotonic function $f: \mathbb{R} \rightarrow \mathbb{R}$ such that $f(x+1)=f(x)+1$ and $C$ is the projection of $G_{f}$. Clearly, we can normalize $f$ so that $f(0)=0$.

It is easy to see that on the interval $[0,1]$ we have $f_{-} \leq f \leq f_{+}$, so the same inequalities hold on the whole real line.

Remark 6.13. The property to be connectible by an achronal meridian is closed. That is, if $\Delta$ is connectible, so is $\bar{\Delta}$.

Remark 6.14. If $\Delta$ is connectible by an achronal meridian and it is connected, then $\Delta$ is itself an achronal meridian and $C_{-}=C_{+}$.

Otherwise we can consider the region $Q(\Delta)$ obtained by projecting to the quotient the region

$$
\tilde{Q}=\left\{(x, y) \in[0,1]^{2} \mid f_{-}(x) \leq y \leq f_{+}(x)\right\}
$$

It is the union of the closure of $\Delta$ and some rectangles whose edges (that are light-like for the conformal structure of $\partial_{\infty} \mathrm{AdS}_{3}$ ) are contained in $G_{f_{-}}$and $G_{f_{+}}$(we consider also 
the degenerate case when two opposite edges collapse to points). Two opposite vertices of such rectangles lie in $\Delta$.

Clearly, every achronal meridian containing $\Delta$ is contained in $Q$. Conversely if for every rectangle, an achronal arc connecting the two vertices in $\Delta$ is chosen, then the closure of the union of such arcs is an achronal meridian.
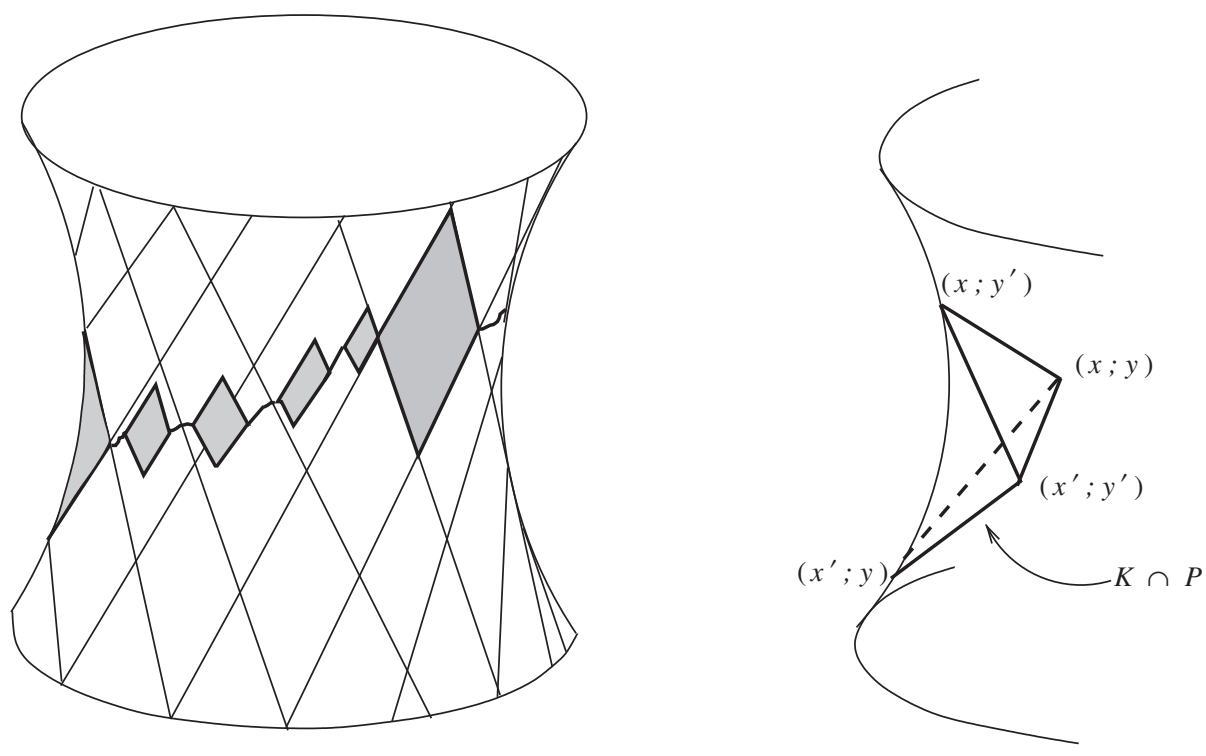

Remark 6.15. Let $\Delta=\left\{\left(x_{i}, y_{i}\right)\right\}$ be connectible by an achronal meridian. Let $I$ be a component of $\partial \mathbb{H}^{2} \backslash \overline{\left\{x_{i}\right\}}$. Then there is a rectangle $R$ in $Q(\Delta) \backslash \Delta$ of the form $I \times J$. Moreover, this is a one-to-one correspondence.

In particular given two points $(x, y),\left(x^{\prime}, y^{\prime}\right) \in \bar{\Delta}$ with $x \neq x^{\prime}$, if there is no point $p=\left(x^{\prime \prime}, y^{\prime \prime}\right)$ in $\Delta$ such that $x<x^{\prime \prime}<x^{\prime}$ then the rectangle $\left[x, x^{\prime}\right] \times\left[y, y^{\prime}\right]$ is contained in $Q(\Delta)$ (here $\left[y, y^{\prime}\right]$ is the positive segment joining $y$ to $y^{\prime}$ if they are different, otherwise $\left[y, y^{\prime}\right]=\{y\}$.

Remark 6.16. Let $\Delta$ be connectible by an achronal meridian and $C_{-}$be the lower meridian through $\Delta$. Consider a non-degenerate rectangle in $Q(\Delta)$, say $R=\left[x, x^{\prime}\right] \times\left[y, y^{\prime}\right]$. Recall that the light-like plane $P$ tangent to $\partial_{\infty} \mathrm{AdS}_{3}$ at a point $\left(x^{\prime}, y\right)$ meets $\partial_{\infty} \mathrm{AdS}_{3}$ along the leaves through $\left(x^{\prime}, y\right)$. It follows that $P$ does not separate $C_{-}$, so it is a support plane for the convex hull $K$ of $C_{-}$. In particular, $K \cap P$ is the light-like ideal triangle with vertices $(x, y),\left(x^{\prime}, y\right),\left(x^{\prime}, y^{\prime}\right)$. 
Let us come back to our original problem. By Lemmas 6.9 and 6.12, we conclude that $\partial_{\infty} \mathcal{H}$ is connectible by an achronal meridian. Let $C_{-}$be the extremal lower meridian passing through $\partial_{\infty} \mathcal{H}$.

Remark 6.17. Since $\partial_{\infty} \mathcal{H}$ is invariant for the representation $\left(h_{\mathrm{l}}, h_{\mathrm{r}}\right)$, it is easy to check that also $C_{-}$is invariant.

We are going to prove that the curve $C_{-}$determines the bent surface $b(\mathcal{H})$ and thus determines the earthquake $E^{r}$. Recall that $b(\mathcal{H})$ is defined in part 5.2.

Proposition 6.18. The bent surface $b(\mathcal{H})$ is the space-like part of the future boundary of the convex hull $K$ of $C_{-}$.

Proof. For every stratum $F$ of the fault lamination, the set $K(F)$ is the convex hull of its ideal points that lie in $\partial_{\infty} \mathcal{H}$. It follows that $b(\mathcal{H})$ is contained in $K$.

We claim that $b(\mathcal{H})$ is contained in the upper boundary of $K$. Given a stratum $F$ of the fault locus, let $P_{F}$ denote the space-like plane in $\operatorname{AdS}_{3}$ containing $K(F)$. We will prove that $P_{F}$ is an upper support plane for $K$.

Indeed, up to post-composition with an element in $P S L_{2}(\mathbb{R})$ we may suppose that $\left.E^{r}\right|_{F}=I d$. It follows that $P_{F}$ is the plane $P_{0}$ whose trace at infinity is the diagonal of $\partial \mathbb{H}^{2} \times \partial \mathbb{H}^{2}$.

Let $F^{\prime}$ be another stratum. There are two ideal points $x, x^{\prime} \in \partial_{\infty} F$ such that the geodesic in $\mathbb{H}^{2}$ with endpoints $x$ and $x^{\prime}$ is a component of the frontier of $F$ and $\partial_{\infty} F^{\prime}$ is contained in the positive interval $\left(x, x^{\prime}\right)$. The fact that $F^{\prime}$ is moved on the right as seen from $F$ means that if $y \in \partial_{\infty} F^{\prime}$ then either $y=E^{r}(y) \in\left\{x, x^{\prime}\right\}$ or $x<E^{r}(y)<y$.

This shows that the pre-image of $\partial_{\infty} \mathcal{H}$ on the square $(0,1) \times(0,1)$ (where we are assuming that the point $(0,0)$ corresponds to an ideal point of $F)$ is contained in the triangle $\{(u, v) \mid v \leq u\}$.

It easily follows that $f_{-}(u) \leq u$ for every $u \in[0,1]$. By Remark 6.5, the plane $P_{F}$ turns out to be an upper support plane for $K$. This shows that $b(\mathcal{H})$ is contained in the space-like part of $\partial_{+} K$.

Let $p \in \partial_{+} K \backslash b(\mathcal{H})$. We will show that a light-like support plane passes through it. Suppose that $P$ is a space-like support plane through $p$. Let us consider $P \cap C_{-}=$ $\left\{\left(x_{i}, y_{i}\right)\right\}$, and let $F_{P}$ be the convex hull in $\mathbb{H}^{2}$ of the points $x_{i}$. By Lemma 6.6 and Remark 6.7, we have that $F_{P}$ is weakly separated by all the strata of the fault lamination. In particular, it is separated from the universal cover, $\mathcal{H}_{l}$, of $\Sigma_{\eta}$. 
It follows that points $x_{i}$ are all contained in some component $I$ of $\partial \mathbb{H}^{2} \backslash \partial \mathcal{H}_{l}$. Thus, $P \cap C_{-}$is contained in the rectangle $R$ of $Q\left(\partial_{\infty} \mathcal{H}\right)$ corresponding to the interval $I$. $R \cap C_{-}$is the union of the two lower edges connecting vertices $q, q^{\prime} \in \overline{\partial_{\infty} \mathcal{H}}$.

Using the fact that $P$ is a space-like support plane, it follows easily that $P \cap C_{-}=$ $\left\{q, q^{\prime}\right\}$, thus $p$ lies on the geodesic with endpoints $q, q^{\prime}$.

By Remark 6.16, the light-like plane tangent to $\partial_{\infty} \mathrm{AdS}_{3}$ at the lower vertex of $R$ is a support plane for $K$ containing $q, q^{\prime}$ and thus $p$. It follows that $p$ is not contained in the space-like region of $\partial_{+} K$.

\section{The Action of $\pi_{1}(\Sigma)$ on $\partial \mathbb{H}^{2} \times \partial \mathbb{H}^{2}$}

Given two elements $\eta_{l}, \eta_{r} \in \mathcal{T}_{g, n}$ with corresponding holonomies $h_{1}, h_{\mathrm{r}}: \pi_{1}(\Sigma) \rightarrow$ $P S L(2, \mathbb{R})$, let us consider the action of the product representation

$$
h=\left(h_{1}, h_{\mathrm{r}}\right): \pi_{1}(\Sigma) \rightarrow P S L(2, \mathbb{R}) \times P S L(2, \mathbb{R})
$$

on $\partial \mathbb{H}^{2} \times \partial \mathbb{H}^{2}$. Since neither $h_{1}$ nor $h_{\mathrm{r}}$ fixes a point in $\partial \mathbb{H}^{2}, \pi_{1}(\Sigma)$ fixes no point on $\partial \mathbb{H}^{2} \times$ $\partial \mathbb{H}^{2}$.

Given $\gamma \in \Gamma$, denote by $x_{L}^{ \pm}(\gamma)$ (resp. $x_{R}^{ \pm}(\gamma)$ ) the attractive and repulsive fixed points of $h_{1}(\gamma)$ (resp. $h_{\mathrm{r}}(\gamma)$ ). If $h_{1}(\gamma)$ is parabolic, then $x_{L}^{+}(\gamma)=x_{L}^{-}(\gamma)$ is the unique fixed point of $h_{1}(\gamma)$. Let us introduce the following notations for the fixed points of $h(\gamma)$ :

$$
\begin{aligned}
& p_{++}(\gamma)=\left(x_{L}^{+}(\gamma), x_{R}^{+}(\gamma)\right), p_{+-}(\gamma)=\left(x_{L}^{+}(\gamma), x_{R}^{-}(\gamma)\right), \\
& p_{-+}(\gamma)=\left(x_{L}^{-}(\gamma), x_{R}^{+}(\gamma)\right), p_{--}(\gamma)=\left(x_{L}^{-}(\gamma), x_{R}^{-}(\gamma)\right) .
\end{aligned}
$$

For every $\alpha, \gamma \in \pi_{1}(S)$, the following identities hold

$$
\begin{aligned}
& p_{++}\left(\gamma^{-1}\right)=p_{--}(\gamma), \quad p_{+-}\left(\gamma^{-1}\right)=p_{-+}(\gamma) \\
& p_{ \pm \pm}\left(\alpha \gamma \alpha^{-1}\right)=h(\alpha) p_{ \pm \pm}(\gamma)
\end{aligned}
$$

It is also easy to see that for every $p \in \partial \mathbb{H}^{2} \times \partial \mathbb{H}^{2} \backslash\left\{p_{-+}(\gamma), p_{+-}(\gamma), p_{--}(\gamma)\right\}$

$$
\lim _{k \rightarrow+\infty} h\left(\gamma^{k}\right)(p)=p_{++}(\gamma)
$$

A consequence of the last fact is that any non-empty $h$-invariant closed subset of $\partial \mathbb{H}^{2} \times \partial \mathbb{H}^{2}$ must contain $p_{++}(\gamma)$ for every $\gamma \in \pi_{1}(\Sigma)$. 
Definition 7.1. We define:

$$
\Lambda=\Lambda\left(h_{1}, h_{\mathrm{r}}\right)=\overline{\left\{p_{++}(\gamma) \mid \gamma \in \pi_{1}(\Sigma)\right\}}
$$

By the remarks above, $\Lambda$ is the smallest non-empty closed $h$-invariant subset of $\partial \mathbb{H}^{2} \times \partial \mathbb{H}^{2}$.

We state in the next proposition some basic properties of $\Lambda$, we sketch the proof referring to $[17,2,3,4]$ for details.

\section{Proposition 7.2.}

(1) The projection on the first factor, $\pi_{L}: \partial \mathbb{H}^{2} \times \partial \mathbb{H}^{2} \rightarrow \partial \mathbb{H}^{2}$, sends $\Lambda$ on to the limit set of $h_{1}$.

(2) If both $\eta_{l}$ and $\eta_{r}$ are complete (i.e., with only cusps), then $\Lambda$ is the graph of the homeomorphism of $f: \partial \mathbb{H}^{2} \rightarrow \partial \mathbb{H}^{2}$ conjugating $h_{\mathrm{l}}$ and $h_{\mathrm{r}}$. Otherwise, $\Lambda$ is a Cantor set.

(3) The metrics $\eta_{l}$ and $\eta_{r}$ are isotopic if and only if $\Lambda$ is contained in the boundary of a space-like plane.

Proof. Notice that $\pi_{L}(\Lambda)$ is a closed subset of $\partial \mathbb{H}^{2}$ invariant under $h_{\mathrm{l}}$. Thus, it contains the limit set, say $\Lambda_{l}$, of $h_{1}$.

On the other hand, $\pi_{L}^{-1}\left(\Lambda_{l}\right)$ is a closed subset of $\partial \mathbb{H}^{2}$ invariant under $h$. So it must contain $\Lambda$.

Thus, we have proved

- $\Lambda_{l} \subset \pi(\Lambda)$

- $\Lambda \subset \pi_{L}^{-1}\left(\Lambda_{l}\right)$

and we can conclude that $\Lambda_{l}=\pi_{L}(\Lambda)$.

For point (2), let $f$ be the equivariant homeomorphism of $\partial \mathbb{H}^{2}$ conjugating $h_{1}$ with $h_{\mathrm{r}}$. Since the graph of $f$ is invariant by $h$, it contains $\Lambda$. On the other hand, since $\pi_{L}(\Lambda)=\Lambda_{l}=\partial \mathbb{H}^{2}$, it follows that $\Lambda$ coincides with the whole graph.

Finally for the third point, notice that if $h_{1}$ and $h_{\mathrm{r}}$ are conjugated in $P S L_{2}(\mathbb{R})$, then up to conjugation the points $p_{++}(\gamma)$ lie all on the diagonal of $\partial \mathbb{H}^{2} \times \partial \mathbb{H}^{2}$. It follows that $\Lambda$ is contained in the boundary of $P_{0}$. 
On the other hand, if $\Lambda$ is contained in the boundary of some space-like plane $P_{A}$ then for every $\gamma \in \pi_{1}(S)$ we have

$$
x_{R}^{+}(\gamma)=A x_{L}^{+}(\gamma)
$$

It follows that for every $\gamma$ the attractive and repulsive fixed points of $h_{\mathrm{l}}(\gamma)$ and $A^{-1} h_{\mathrm{r}}(\gamma) A$ coincide. Thus, we conclude that

$$
h_{\mathrm{l}}(\gamma)=A^{-1} h_{\mathrm{r}}(\gamma) A
$$

The action of $\pi_{1}(\Sigma)$ on $\partial \mathbb{H}^{2} \times \partial \mathbb{H}^{2}$ is reminiscent of a quasifuchsian action of $\pi_{1}(\Sigma)$ on $S^{2}=\partial_{\infty} \mathbb{H}^{3}$. An important difference with that case is that the action of $\pi_{1}(\Sigma)$ on $\partial \mathbb{H}^{2} \times \partial \mathbb{H}^{2} \backslash \Lambda$ is not proper. Indeed, $p_{+-}(\gamma)$ is not contained in $\Lambda$ and is fixed by $h(\gamma)$. We are going to describe a maximal $h$-invariant domain of $\partial \mathbb{H}^{2} \times \partial \mathbb{H}^{2}$, on which the action is properly discontinuous and causal. This set could be regarded as the analogous of the discontinuity domain.

Given an element $\gamma \in \pi_{1}(S)$ parallel to a puncture, we consider the two intervals $I_{L}(\gamma)$ and $I_{R}(\gamma)$ in $\partial \mathbb{H}^{2}$ that corresponds to the infinite end of $\gamma$. If $h_{\mathrm{l}}(\gamma)$ (resp. $h_{\mathrm{r}}(\gamma)$ ) is parabolic, then $I_{L}(\gamma)$ (resp. $\left.I_{R}(\gamma)\right)$ is reduced to a point.

Proposition 7.3. The set $\Lambda$ is connectible by an achronal meridian.

The region, say $\mathcal{G}$, between the upper and lower meridians passing through $\Lambda$ is

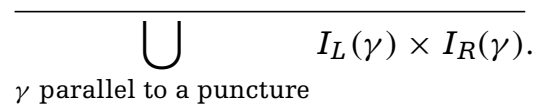

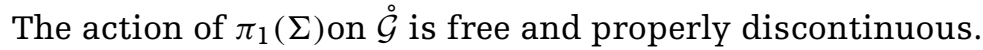

Proof. Let $\Lambda_{l}^{*}$ be the set of conical limit points of $h_{\mathrm{l}}$. Let $f: \mathcal{H}_{l} \rightarrow \mathcal{H}_{r}$ be the lifting of some orientation-preserving diffeomorphism $f: \Sigma_{\eta_{l}} \rightarrow \Sigma_{\eta_{r}}$. We know that $f$ extends to a continuous map

$$
f: \mathcal{H}_{l} \cup \Lambda_{l}^{*} \rightarrow \mathcal{H}_{r} \cup \partial \mathbb{H}^{2}
$$

sending $\Lambda_{l}^{*}$ to some subset of $\Lambda_{r}$.

It is easy to see that the graph of $\left.f\right|_{\Lambda_{l}^{*}}$ is contained in $\Lambda$. We call this set $\Lambda^{*}$. Since it is invariant under $h$, its closure is $\Lambda$. 
So by Remark 6.13 , it is sufficient to prove that $\Lambda^{*}$ is connectible by an achronal meridian.

Now let us take $x_{1}<x_{2}<x_{3} \in \Lambda_{l}^{*}$ and suppose $f\left(x_{1}\right) \neq f\left(x_{3}\right)$. Consider the oriented geodesic $l$ joining $x_{1}$ to $x_{3}$ and let $r$ be the half-line joining a point of $l$ to $x_{2}$. By our assumption, $r$ is contained on the right side bounded by $l$.

It follows that $f(r)$ is contained on the right side of $f(l)$. On the other hand, $f(l)$ has first endpoint $f\left(x_{1}\right)$ and $f(r)$ joins a point of $f(l)$ to $f\left(x_{2}\right)$. So $f\left(x_{1}\right) \leq f\left(x_{2}\right) \leq f\left(x_{3}\right)$.

To conclude, we have to show that $\mathcal{G}$ is the closure of the union of $I_{L}(\gamma) \times I_{R}(\gamma)$.

By Remark 6.15, there is a one-to-one correspondence between rectangles of $\mathcal{G} \backslash$ $\Lambda$ and components of $\partial \mathbb{H}^{2} \backslash \Lambda_{l}$. Every component of $\partial \mathbb{H}^{2} \backslash \Lambda_{l}$ is of form $I_{L}(\gamma)$ for some peripheral element $\gamma$.

Let $G$ be the rectangle in $\mathcal{G}$ corresponding to the interval $I_{L}(\gamma) \times I_{R}(\gamma)$. Since the interior of $I_{L}(\gamma) \times I_{R}(\gamma)$ does not contain points of $\Lambda$, by Remark 6.15 we have that $I_{L}(\gamma) \times I_{R}(\gamma)$ is contained in $G$.

On the other hand, notice that $p_{--}(\gamma)=\left(x_{-}^{L}(\gamma), x_{-}^{R}(\gamma)\right)$ and $p_{++}(\gamma)=$ $\left(x_{+}^{L}(\gamma), x_{+}^{R}(\gamma)\right)$ are both in $\Lambda$. In particular, this shows that if $C$ is a meridian curve containing $\Lambda$ then $C \cap \pi_{L}^{-1}\left(I_{L}(\gamma)\right)$ is contained in $I_{L}(\gamma) \times I_{R}(\gamma)$.

This shows that $G \subset I_{L}(\gamma) \times I_{R}(\gamma)$ and the proof is complete.

We say that a $\pi_{1}(\Sigma)$-invariant achronal meridian is extremal if it is contained in the boundary of $\mathcal{G}$.

Remark 7.4. The number of non-degenerate rectangles $I_{L}(\gamma) \times I_{R}(\gamma)$ up to the action of $\pi_{1}(\Sigma)$ is equal to the number, say $k$, of punctures of $\Sigma$ that corresponds to boundary components for both $\Sigma_{\eta_{l}}$ and $\Sigma_{\eta_{r}}$.

It follows from Remark 6.14 that there are exactly $2^{k}$ extremal $\pi_{1}(\Sigma)$-invariant meridians.

\section{Earthquakes and Extremal Invariant Curves}

In this section, we clarify the relation between earthquakes on a hyperbolic surface with geodesic boundary and extremal curves on the boundary at infinity $\mathrm{AdS}_{3}$ which are invariant under the action of a group. This will lead in particular to the proof of Theorem 1.2. 


\subsection{Bent surfaces constructed from an earthquake}

Let us fix two admissible metrics $\eta_{l}$ and $\eta_{r}$. Let

$$
h=\left(h_{1}, h_{\mathrm{r}}\right): \pi_{1}(\Sigma) \rightarrow P S L_{2}(\mathbb{R}) \times P S L_{2}(\mathbb{R})
$$

be the representation whose components are the holonomies of $\eta_{l}$ and $\eta_{r}$, respectively.

Let $\lambda$ be a measured geodesic lamination on $\Sigma_{\eta_{l}}$ such that $E_{\lambda}^{r}\left(\Sigma_{\eta_{l}}\right)=\Sigma_{\eta_{r}}$. Consider the lifted earthquakes $\tilde{E}: \tilde{\Sigma}_{\eta_{l}} \rightarrow \tilde{\Sigma}_{\eta_{r}}$ and let

$$
b: \mathcal{H} \rightarrow \mathrm{AdS}_{3}
$$

be the admissible $\pi_{1}(\Sigma)$-invariant past bent surface constructed as in Section 5.2.

We denote by $S_{+}$the quotient of $\mathcal{H}$ by the action of $\pi_{1}(\Sigma)$. By Proposition 5.7, $S_{+}=E_{\lambda / 2}^{r}\left(\Sigma_{\eta l}\right)$.

Proposition 8.1. The lower meridian passing through $\partial_{\infty} \mathcal{H}$, say $C$, is an extremal $\pi_{1}(\Sigma)$ invariant meridian.

Proof. Given an element $\gamma \in \pi_{1}(\Sigma)$ corresponding to a geodesic boundary for both $\eta_{l}$ and $\eta_{r}$, let us set $G=I_{L}(\gamma) \times I_{R}(\gamma)$. We have to show that $\partial_{\infty} \mathcal{H}$ does not intersect $\stackrel{\circ}{G}$.

Given a component $F$ of $\tilde{\Sigma}_{\eta} \backslash \lambda$, the corresponding stratum of the bent surface $b(\mathcal{H})$, say $K(F)$, is the ideal polygon in $\mathrm{AdS}_{3}$ whose endpoints are the pairs $(x, A x)$ where $x$ is an ideal point of $F$ and $A \in P S L_{2}(\mathbb{R})$ is determined by requiring that $\left.\tilde{E}\right|_{F}=\left.A\right|_{F}$.

Since $F$ is contained in the convex hull of $\Lambda_{l}$, no ideal point of $F$ is contained in $\stackrel{\circ}{I}_{L}(\gamma)$. It turns out that $K(F) \cap \stackrel{\circ}{G}=\emptyset$.

Let us describe more precisely the curve $C$. In particular, we will describe for every region $G$ the intersection $G \cap C$.

Proposition 8.2. Let $\gamma \in \pi_{1}(\Sigma)$ be the peripheral loop corresponding to a geodesic boundary for both $\eta_{l}$ and $\eta_{r}$. Let $m$ be the total mass of $\lambda$ around $p$ and $a$ be the length of the boundary component of $S_{+}$corresponding to $\gamma$ ( $a=0$ if $\gamma$ corresponds to a cusp in $S_{+}$). Then $G \cap C$ is the lower curve if $m \leq a$, is the upper curve otherwise. 
Proof. Let $h_{+}$be the holonomy of $S_{+}$. We can choose coordinates on $\mathbb{H}^{2}$-considered in the Poincaré half-plane model-in such a way that $h_{+}(\gamma) z=e^{2 a} z\left(h_{+}(\gamma) z=z+1\right.$ if $\left.a=0\right)$. In particular, if $a \neq 0$ we can suppose that $\tilde{F} \subset\{z \in \mathbb{C} \mid \operatorname{Re}(z)<0\}$.

If $m \neq 0$, we have two possibilities: leaves near $p$ lift to leaves with endpoints either at 0 or at $\infty$ (if $a=0$ only the last possibility holds, in the other cases the choice depends on the way of spiraling of $\lambda$ around $p$ ). A hyperbolic transformation with attractive fixed point at $\infty$ (resp. 0) is upper triangular (resp. lower triangular).

Thus if we choose a base point near the puncture, it is easy to see that if leaves of $\lambda$ near $p$ lift to geodesics with an endpoint at $\infty$, then as in Proposition 3.3 we have

$$
h_{\mathrm{l}}(\gamma)=\left(\begin{array}{cc}
e^{a-m} & * \\
0 & e^{-(a-m)}
\end{array}\right) h_{\mathrm{r}}(\gamma)=\left(\begin{array}{cc}
e^{a+m} & * \\
0 & e^{-(a+m)}
\end{array}\right) \text {. }
$$

In the same way, if the common endpoint of these geodesics is 0 then we have

$$
h_{\mathrm{l}}(\gamma)=\left(\begin{array}{cc}
e^{(a+m)} & 0 \\
* & e^{-(a+m)}
\end{array}\right) h_{\mathrm{r}}(\gamma)=\left(\begin{array}{cc}
e^{a-m} & 0 \\
* & e^{-(a-m)}
\end{array}\right) .
$$

Since $h_{\mathrm{l}}(\gamma)$ and $h_{\mathrm{r}}(\gamma)$ are assumed to be hyperbolic, we find that $a \neq m$.

Let us distinguish three cases.

(1) $m=0$ : in this case $\gamma$ corresponds to a boundary component of $F$ and the bending map

$$
\beta: \tilde{F} \rightarrow \mathrm{AdS}_{3}
$$

extends to the axis of $\gamma$, say $l$. Moreover, the image of $l$ is the axis of $(\gamma)$ (i.e., the geodesic joining the limit endpoints of $G$ ). Thus, $\partial F_{\lambda} \cap G$ is the past extremal curve.

(2) $0<m<a$. Let $l_{0}$ be a leaf of $\tilde{\lambda}$ with an endpoint fixed by $\gamma$, say $s_{0} \in\{0, \infty\}$. Denote by $t_{0} \in \mathbb{R}_{<0}$ its other endpoint. Notice that the image of $\gamma^{k}\left(l_{0}\right)$ through $\beta$ is a geodesic, say $\hat{l}_{k}$, of $\mathrm{AdS}_{3}$, with endpoints equal to $\left(s_{0}, s_{0}\right)$ and to $\left(h_{1}^{k}(\gamma) t_{0}, h_{\mathrm{r}}^{k}(\gamma) t_{0}\right)$.

Notice that $\left(h_{1}^{k}(\gamma) t_{0}, h_{\mathrm{r}}^{k}(\gamma) t_{0}\right)$ converges to $p_{++}(\gamma)$ as $k \rightarrow+\infty$ and to $p_{--}(\gamma)$ as $k \rightarrow-\infty$. On the other hand, since $m<a$ we have that $\left(s_{0}, s_{0}\right)=p_{++}(\gamma)$ if $s_{0}=\infty$ and $\left(s_{0}, s_{0}\right)=p_{--}(\gamma)$ if $s_{0}=0$. 
Suppose for the sake of simplicity that $s_{0}=\infty$. Then the geodesic $\hat{l}_{k}$ converges to $\left(s_{0}, s_{0}\right)$ as $k \rightarrow+\infty$, but $\hat{l}_{k}$ converges to the geodesic $c_{\gamma}$ with endpoints $p_{--}(\gamma)$ and $p_{++}(\gamma)$ as $k \rightarrow-\infty$. It follows that such a geodesic is contained in the boundary of $\mathcal{H}$. Then, as in the previous case, we get that $\partial F_{\lambda} \cap G$ is the past extremal curve.

(3) $m>a$. In this case $\left(s_{0}, s_{0}\right) \in\left\{p_{-+}(\gamma), p_{+-}(\gamma)\right\}$. Thus, by arguing as before, we get that $\hat{l}_{k}$ converges to the light-like segment in $\partial_{\infty} \mathrm{AdS}_{3}$ joining $p_{++}(\gamma)$ to $\left(s_{0}, s_{0}\right)$ as $k \rightarrow+\infty$ and converges to the light-like segment in $\partial_{\infty} \mathrm{AdS}_{3}$ joining $p_{--}(\gamma)$ to $\left(s_{0}, s_{0}\right)$ as $k \rightarrow-\infty$. Thus, the upper extremal curve of $G(\gamma)$ is contained in the closure of the image of $\beta$ and thus it is contained in $\tilde{F}_{\lambda}$.

Thus, we have proved that if $(F, \lambda)$ encodes a bent surface in $M$, and $F$ is admissible, then the boundary curve of $\tilde{F}_{\lambda}$ is extremal.

\subsection{Constructing an earthquake from an invariant curve}

In this subsection, we prove the following proposition, the last missing tool for the proof of Theorem 1.2.

Proposition 8.3. Let $C$ be an extremal $\pi_{1}(\Sigma)$-invariant achronal meridian and let $K$ be its convex hull. There is an earthquake $E_{\lambda}^{r}$ sending $\Sigma_{\eta_{l}}$ into $\Sigma_{\eta_{r}}$ whose associated admissible bent surface is the space-like part of $\partial_{+} K$.

To prove Proposition 8.3, we need the following simple technical lemma of hyperbolic geometry.

Lemma 8.4. Let $H$ be a closed path-connected subset of $\mathbb{H}^{2}$ and suppose that there exists a decomposition:

$$
H=\bigcup_{i} H_{i}
$$

where each $H_{i}$ is either an ideal geodesic polygon or a geodesic, and $H_{i}$ and $H_{j}$ are weakly separated in $\mathbb{H}^{2}$. Then $H$ is convex.

Proof. Let $c:[0,1] \rightarrow \mathbb{H}^{2}$ be the geodesic segment joining two points $p, q \in H$. Consider the set of $t$ such that $\left.c\right|_{[0, t]}$ is contained in $H$ and let $t_{0}$ the sup of this set. 
By contradiction, suppose $t_{0}<1$ and let $p^{\prime}=c\left(t_{0}\right)$. It is not difficult to construct a family of geodesics $l_{n}$ such that each $l_{n}$ lies in the frontier of some $H_{i}$ and $l_{n}$ intersects $c$ at a point $c\left(t_{n}\right)$ with $t_{n} \rightarrow t_{0}^{-}$. Up to a subsequence, $l_{n}$ converges to a geodesic $l$ that has the property that is weakly separated from all $H_{i}$. Moreover, $p$ and $q$ lie on opposite sides of $l$. Let $U$ be the open half-plane bounded by $l$ and containing $q$.

Thus if $\gamma$ is the path joining $p$ to $q$ in $H$, then there is a time $s_{0}<1$ such that $\gamma\left(s_{0}\right) \in l$ and $\gamma(s)$ lies in $U$ for $s \in\left(s_{0}, 1\right]$. Let us consider the set $L$ of points $s \in\left[s_{0}, 1\right]$ such that $\gamma(s)$ lies in the frontier of some $H_{i}$. Notice that $L$ is closed and that the components of $\left(s_{0}, 1\right] \backslash L$ are contained in the interior. Now if the set $L$ does not accumulate on $s_{0}$, this means that there exists $H_{i}$ containing all the path $\left.\gamma\right|_{\left[s_{0}, s_{0}+\delta\right]}$. Since $H_{i}$ is weakly separated by $l$, it turns out that $H_{i}$ is an ideal geodesic polygon and $l$ is one of its boundary component. But then $c(t) \in H_{i}$ for $t \in\left[t_{0}, t_{0}+\delta^{\prime}\right]$, contradicting the definition of $t_{0}$.

Suppose now that $s_{0}$ is an accumulation point for $L$. There is a $\delta>0$ such that if $s \leq s_{0}+\delta$ and $s \in L$ then $\gamma(s)$ lies in the frontier $l(s)$ of some $H_{i}$ that must intersect $c$ at some point $c(t)$ with $t>t_{0}$, and $t \rightarrow t_{0}^{+}$as $s \rightarrow s_{0}$. We can also suppose that $s_{0}+\delta \in L$ and let us set $\delta^{\prime}$ such that $c\left(t_{0}+\delta^{\prime}\right) \in l\left(s_{0}+\delta\right)$.

The set of points $\hat{L}=\{t \mid c(t) \in l(s)\}$ is a closed subset that accumulates at $s_{0}$. On the other hand, if $I$ is a component of $\left[t_{0}, t_{0}+\delta\right] \backslash L$ then its endpoints lie, respectively, in $l(s)$ and $l\left(s^{\prime}\right)$ and $\left(s, s^{\prime}\right)$ is a component of $\left[s_{0}, s_{0}+\delta\right] \backslash L$. It follows that $l(s)$ and $l\left(s^{\prime}\right)$ are boundary components of some $H_{i}$, but then the segment $C(I)$ is contained in $H_{i}$. This shows that $\left.C\right|_{\left[t_{0}, t_{0}+\delta^{\prime}\right]}$ is contained in $H$ and we get a contradiction.

Proof of Proposition 8.3. Let us consider the set

$$
\mathcal{P}=\left\{A \in P S L_{2}(\mathbb{R}) \mid P_{A} \text { is a support plane for } \partial_{+} K\right\}
$$

If $A \in \mathcal{P}$, then $P_{A} \cap \partial_{+} K$ is the convex hull of some set $\left\{x_{i}, A x_{i}\right\}_{i \in I}=C \cap P_{A}$.

We define the stratum associated to $A$ to be the convex hull, say $F(A)$, in $\mathbb{H}^{2}$ of $\left\{x_{i}\right\}_{i \in I}$. By Remark 6.7, $F(A)$ is weakly separated from $F\left(A^{\prime}\right)$.

By the invariance of $\partial_{+} K$, if $P$ is a support plane for $\partial_{+} K$, then so is $\left(h_{\mathrm{l}}(\gamma), h_{\mathrm{r}}(\gamma)\right)(P)$. Thus, if $A$ lies in $\mathcal{P}$ then so does $\gamma \cdot A=h_{\mathrm{r}}(\gamma) \circ A \circ h_{\mathrm{l}}(\gamma)^{-1}$ and $F(\gamma \cdot A)=$ $h_{\mathrm{l}}(\gamma)(F(A))$. In particular, the set

$$
\mathcal{H}=\overline{\bigcup_{A \in \mathcal{P}} F(A)}
$$

is $h_{1}$-invariant. 
We claim that $\mathcal{H}$ is the convex core of $h_{1}$. First, we prove that $F(A)$ is contained in the convex core for every $A \in \mathcal{P}$. By contradiction, if $F(A)$ is not contained in the convex core there is some ideal point of $F(A)$ that is not contained in $\Lambda_{l}$. Thus, there is some point $(x, y) \in P_{A} \cap C$ such that $x \in I_{l}(\stackrel{\circ}{\gamma})$ for some peripheral $\gamma$. Since $C$ is an extremal meridian, the point $(x, y)$ must lie on the interior of some edge $e$ of $I_{l}(\gamma) \times I_{r}(\gamma)$. Since $P_{A}$ is a support plane for $K$, it cannot disconnect $C$ (more precisely, in a suitable affine chart $C$ is contained in one of the two closed half-spaces bounded by $P_{A}$ ). It follows that $P_{A}$ must contain $e$. Since $P_{A}$ is space-like, this gives a contradiction (space-like planes intersect every leaf only once).

This proves that $\mathcal{H}$ is contained in the convex hull. To prove the reverse inclusion, we only have to check that $\mathcal{H}$ is convex.

By Lemma 8.4, it is sufficient to prove that $\mathcal{H}$ is path connected. Notice that by definition, $r_{A}\left(F_{A}\right)=P_{A} \cap \partial_{+} K$. So given two points $p \in F(A)$ and $q \in F\left(A^{\prime}\right)$, let us consider the corresponding points $\hat{p}=r_{A}(p)$ and $\hat{q}=r_{A}(q)$ in $\partial_{+} K$. By classical facts on convex subset in $\mathbb{R}^{3}$, there is a continuous path

$$
u:[0,1] \rightarrow T\left(\mathrm{AdS}_{3}\right), \quad u(t)=(\hat{p}(t), v(t))
$$

such that $\hat{p}(t)$ is a path in the space-like part of $\partial_{+} K$ joining $\hat{p}$ to $\hat{q}$ and $v(t)$ is a vector orthogonal to some support plane $P(t)$ at $\hat{p}(t)$ of $\partial_{+} K$.

Let $A(t) \in P S L_{2}(\mathbb{R})$ be such that $P(t)=P_{A(t)}$. Then the path

$$
p(t)=r_{A(t)}^{-1} p(t)
$$

is a continuous path in $\mathbb{H}^{2}$ joining $p$ to $q$. Since $p(t) \in F(A(t))$, we conclude that $p$ and $q$ are connected by an arc in $\mathcal{H}$, so it is connected.

We consider on $\mathcal{H}$ the geodesic lamination

$$
L=\bigcup_{A \in \mathcal{P}: F(A) \text { is a geodesic }} F(A) \cup \bigcup_{A \in \mathcal{P}: F(A) \text { is a polygon }} \partial F(A)
$$

We construct a right earthquake on $\mathcal{H}$ with fault locus $L$ and such that the corresponding bent surface is $\partial_{+} K$. 
Indeed, every stratum $F$ of $L$ coincides with $F(A)$ for some $A \in \mathcal{P}$. So we select for every stratum $F$ an element $A=A(F) \in \mathcal{P}$ such that $F=F(A)$ (the choice is unique almost everywhere). So we define the map

$$
E: \mathcal{H} \rightarrow \mathbb{H}^{2}
$$

such that $\left.E\right|_{F}=A(F)$.

Let us consider two strata $F$ and $F^{\prime}$. The planes $P_{A(F)}$ and $P_{A\left(F^{\prime}\right)}$ meet along a line $l$. Orient $l$ in such a way that the signed angle between $P_{A(F)}$ and $P_{A\left(F^{\prime}\right)}$ is positive.

Let $\left(x_{-}, y_{-}\right)$and $\left(x_{+}, y_{+}\right)$be the endpoints of $l$. Since $y_{ \pm}=A(F) x_{ \pm}=A\left(F^{\prime}\right) x_{ \pm}$, it turns out that $x_{-}$and $x_{+}$are fixed points for the comparison isometry $B^{*}=A(F)^{-1} \circ$ $A\left(F^{\prime}\right)$.

Thus, $B^{*}$ is a hyperbolic translation whose axis is the geodesic $s$ in $\mathbb{H}^{2}$ with endpoints $x_{-}$and $x_{+}$. From Remark 6.7, $s$ separates $F$ from $F^{\prime}$. So in order to conclude, we just have to check that $F^{\prime}$ is moved by $B^{*}$ on the right as viewed from $F$. Since $F^{\prime}$ is contained in the left side bounded by $s$, it is sufficient to prove that $B^{*}$ acts by a positive translation on $s$.

Notice that the isometry $\left(B^{*}, 1\right)$ sends $P_{A\left(F^{\prime}\right)}$ into $P_{A(F)}$. Since the signed angle $\alpha\left(P_{A\left(F^{\prime}\right)}, P_{A(F)}\right)<0$, then the rotation component of $\left(B^{*}, 1\right)$ is negative. By Lemma 5.4 , we conclude that $t\left(B^{*}\right)>0$.

By applying construction of Proposition 6.18 to the earthquake $E$, we easily check that the bent surface associated to $E$ is the space-like part of $\partial_{+} K$.

\subsection{Proof of the main result}

We are now ready to prove the Earthquake Theorem for hyperbolic surfaces with geodesic boundary.

Proof of Theorem 1.2. Proposition 8.3, along with Proposition 8.1, shows that there is a one-to-one correspondence between right earthquakes relating $\eta_{l}$ to $\eta_{r}$ and extremal curves. According to Remark 7.4, the number of extremal curves is equal to $2^{k}$, where $k$ is the number of boundary components which are cusps neither for $\eta_{l}$ nor for $\eta_{r}$. Theorem 1.2 follows.

\section{The Enhanced Teichmüller Space}

The enhanced Teichmüller space $\hat{\mathcal{T}}_{g, n}$ of a compact surface with boundary is defined in the introduction (Definition 1.3). There is a natural topology on $\hat{\mathcal{T}}_{g, n}$, which restricts to 
the domains where all $\epsilon_{i}$ are constant as the usual topology on $\mathcal{T}_{g, n}$. It can be defined through a family of neighborhoods of a point, involving quasiconformal homeomorphisms diffeomorphic to the identity, as well of course as the $\epsilon_{i}$ (we leave the details to the reader).

We now wish to define earthquakes as maps from $\hat{\mathcal{T}}_{g, n}$ to itself. A naive possibility would be to define it as the earthquakes on $\mathcal{T}_{g, n}$, adding some information on the signs assigned to boundary components. This however would yield a definition which is not quite satisfactory, since right earthquakes would not have some desirable properties, like those appearing in Proposition 9.7 below.

Reflections of geodesic laminations. Some preliminary definitions are needed. Here we consider a compact surface $S$ of genus $g$ with $n$ boundary components, and a hyperbolic metric $h$ with geodesic boundary on $S$. Let $c_{0}$ be one of the boundary components of $S$ which is not a cusp. Let $\gamma$ be a complete oriented embedded geodesic in $(S, h)$ which is asymptotic to $c_{0}$ on its positive endpoint, that is, which spirals on to $c_{0}$ as $t \rightarrow \infty$.

Definition 9.1. The reflection of $\gamma$ relative to $c_{0}$, denoted by $\sigma_{c_{0}}(\gamma)$, is the geodesic in $(S, h)$ obtained as follows. Let $\bar{\gamma}$ be any lift of $\gamma$ to the universal cover $\tilde{S}$ of $S$, so that $\bar{\gamma}$ has its endpoint on the positive side at an endpoint of a lift $\bar{c}_{0}$ of $c_{0}$. We define $\sigma_{c_{0}}(\gamma)$ to be the projection on $S$ of the complete geodesic having as its positive endpoints the other endpoint of $\bar{\gamma}$ and the other endpoint of $\bar{c}_{0}$.

Note that considering an oriented geodesic here is necessary only if $\gamma$ spirals on $c_{0}$ at both ends. The existence of this reflected geodesic can also be considered in light of Lemma 2.3 .

\section{Remarks 9.2.}

(1) $\rho_{c_{0}}(\gamma)$ is also embedded,

(2) if $\gamma_{1}$ and $\gamma_{2}$ are two geodesics asymptotic to $c_{0}$ which are disjoint, then $\rho_{c_{0}}\left(\gamma_{1}\right)$ and $\rho_{C_{0}}\left(\gamma_{2}\right)$ are also disjoint,

(3) if $\gamma_{1}$ is a geodesic asymptotic to $c_{0}$ and $\gamma_{2}$ is a geodesic not asymptotic to $c_{0}$, and if $\gamma_{1}$ and $\gamma_{2}$ are disjoint, then $\sigma_{c_{0}}\left(\gamma_{1}\right)$ and $\gamma_{2}$ are disjoint. 
Proof. For the first point, let $\left(\gamma_{t}\right)_{t \in(0,1)}$ be a one-parameter family of geodesic rays starting from a point of $c_{0}$ and ending at the common endpoint of $\gamma$ and $\sigma_{c_{0}}(\gamma)$, such that $\lim _{t \rightarrow 0} \gamma_{t}=\gamma, \lim _{t \rightarrow 1} \gamma_{t}=\sigma_{C_{0}}(\gamma)$. Since $\gamma_{0}=\gamma$ is embedded, it is not difficult to show that $\gamma_{t}$ is embedded for $t$ small enough. Suppose that $\gamma_{t}$ is not embedded for some $t \in(0,1)$, and let $t_{0}$ be the infimum of the $t \in(0,1)$ such that $\gamma_{t}$ is not embedded, then $\gamma_{t_{0}}$ would have a self-tangency point, which is impossible. So $\gamma_{t}$ is embedded for all $t \in(0,1)$, and therefore $\gamma_{1}=\sigma_{C_{0}}(\gamma)$ is also embedded. This proves the first point.

For the second point, let $\bar{\gamma}_{1}$ and $\bar{\gamma}_{2}$ be any lifts of $\gamma_{1}$ and $\gamma_{2}$ to $\tilde{S}$. Since $\gamma_{1}$ and $\gamma_{2}$ are disjoint, their lifts $\bar{\gamma}_{1}$ and $\bar{\gamma}_{2}$ are also disjoint. $\sigma_{c_{0}}\left(\gamma_{1}\right)$ is the image by the projection $\tilde{S} \rightarrow S$ of the geodesic, which we can denote by $\sigma_{C_{0}}\left(\gamma_{1}\right)$, which has one endpoint in common with $\bar{\gamma}_{1}$ (not on a lift of $c_{0}$ ) while the other is an endpoint of a lift of $c_{0}$ which has as its other endpoint an endpoint of $\bar{\gamma}_{1}$. The same description holds for $\bar{\gamma}_{2}$. It follows that $\sigma_{C_{0}}\left(\bar{\gamma}_{1}\right)$ and $\sigma_{C_{0}}\left(\bar{\gamma}_{2}\right)$ are also disjoint. Since this is true for all lifts of $\gamma_{1}$ and $\gamma_{2}$ to $\tilde{S}, \sigma_{C_{0}}\left(\gamma_{1}\right)$ and $\sigma_{c_{0}}\left(\gamma_{2}\right)$ are disjoint, which proves the second point.

The third point can be proved using the same argument, we leave the details to the reader.

Definition 9.3. Let $\lambda$ be a measured geodesic lamination on $(S, h)$. The reflection of $\lambda$ is the measured lamination, denoted by $\sigma_{C_{0}}(\lambda)$, obtained by replacing each leave of $\lambda$ which is asymptotic to $c_{0}$ by its reflection relative to $c_{0}$.

The previous remarks make this definition possible, since they show that $\sigma_{C_{0}}(\lambda)$ is again a measured geodesic lamination (its support is a disjoint union of geodesics). Note that the reflection map, acting on measured geodesic laminations, has some simple properties:

- if $\lambda$ is any measured geodesic laminations on $S$ then $\sigma_{C_{0}}^{2}(\lambda)=\lambda$,

- if $c_{0}$ and $c_{1}$ are two boundary components of $S$ then $\sigma_{C_{0}}$ and $\sigma_{C_{1}}$ commute.

In both cases, the proofs follow by considering the corresponding statements for geodesics.

Earthquakes on the enhanced Teichmüller space. The definition of earthquakes on $\hat{\mathcal{T}}_{g, n}$ is based on the reflection of measured geodesic laminations.

First, we define the earthquake on the subset of $\hat{\mathcal{T}}_{g, n}$, say $\hat{\mathcal{T}}_{g, n}^{\prime}$ of admissible metrics without cusps. 
Definition 9.4. The map $E_{\mathrm{r}}: \mathcal{M} \mathcal{L}_{g, n} \times \hat{\mathcal{T}}_{g, n}^{\prime} \rightarrow \hat{\mathcal{T}}_{g, n}$ is defined as follows. Let $\left(\eta, \epsilon_{1}, \cdots, \epsilon_{n}\right) \in \hat{\mathcal{T}}_{g, n^{\prime}}^{\prime}$ and let $\lambda \in \mathcal{M L}_{g, n}$. Consider the measured lamination $\bar{\lambda}$ obtained by taking the reflection of $\lambda$ with respect to all boundary components $c_{i}$ of $S$ for which $\epsilon_{i}=-1$, and let $\bar{\eta}=E_{\mathrm{r}}(\bar{\lambda})(\eta)$. Finally, for $i=1, \cdots, n$, let $\bar{\epsilon}_{i}=\epsilon_{i}$ if the right earthquake $E_{\mathrm{r}}(\bar{\lambda})$ does not change the direction in which $\bar{\lambda}$ spirals into $c_{i}, \bar{\epsilon}_{i}=0$ if $c_{i}$ becomes a cusp and $\bar{\epsilon}_{i}=-\epsilon_{i}$ otherwise. Then $E_{\mathrm{r}}(\lambda)\left(\eta, \epsilon_{1}, \cdots, \epsilon_{n}\right)=\left(\bar{\eta}, \bar{\epsilon}_{1}, \cdots, \bar{\epsilon}_{n}\right)$.

The following lemma ensures that it is possible to extend $E_{\mathrm{r}}$ to the whole $\hat{\mathcal{T}}_{g, n}$.

Lemma 9.5. For any $\lambda \in \mathcal{M} \mathcal{L}_{g, n}$, the map $E_{\mathrm{r}}(\lambda): \hat{\mathcal{T}}_{g, n}^{\prime} \rightarrow \hat{\mathcal{T}}_{g, n}$ extends continuously on $\hat{\mathcal{T}}_{g, n}$.

Proof. Given a point $\left(\eta, \epsilon_{1}, \ldots, \epsilon_{k}\right)$ corresponding to a metric $\eta$ with $n-k$ cusps, denote by $\lambda^{\prime}$ the measured geodesic lamination of $\mathcal{M} \mathcal{L}_{g, n}(\eta)$ corresponding to $\lambda$. Let $\bar{\lambda}^{\prime}$ be the lamination obtained by reflecting $\lambda^{\prime}$ with respect to all boundary components for which $\epsilon_{i}=-1$ and let $\bar{\eta}=E_{\mathrm{r}}\left(\bar{\lambda}^{\prime}\right)(\eta)$. For $i=1, \ldots, k$, the sign $\bar{\epsilon}_{i}$ is defined as in the previous case, whereas for $i=k+1, \ldots, n$ the sign $\bar{\epsilon}_{i}=1$ if the lamination $\lambda$ (i.e., a lamination of a surface without cusp) spirals in the positive way with respect to the standard spiraling orientation, and $\epsilon_{i}=-1$ otherwise. Finally, let us define

$$
E_{\mathrm{r}}(\lambda)(\eta)=\left(\bar{\eta}, \bar{\epsilon}_{1}, \ldots, \bar{\epsilon}_{n}\right)
$$

It is clear that the composition

$$
\hat{\mathcal{T}}_{g, n} \stackrel{E_{\mathrm{r}}(\lambda)}{\longrightarrow} \hat{\mathcal{T}}_{g, n} \stackrel{\pi}{\longrightarrow} \mathcal{T}_{g, n}
$$

is continuous. To conclude, it is then sufficient to show the following points:

(1) if $c_{i}$ is a cusp with respect to $\eta_{0}$, then there is a neighborhood of $\eta$ such that $\bar{\epsilon}_{i}$ is constant.

(2) if $c_{i}$ is a cusp with respect to $E_{\mathrm{r}}(h)\left(\eta_{0}\right)$, then for $a>0$ there is a neighborhood of $\eta_{0}$ such that the length of $c_{i}$ with respect $E_{\mathrm{r}}(\hat{\lambda})(\eta)$ is smaller than $a$ for $\eta$ in that neighborhood.

The second point follows from the continuity of (4). For the first point, let $\varepsilon=1$ if $\lambda$ spirals in the positive way around $c_{i}$ and $\varepsilon=-1$ otherwise. Notice that in a small neighborhood $U$ of $\eta_{0}$ (precisely the set of $\eta$ for which the length of $c_{i}$ is less than the $\lambda$ 
mass of $c_{i}$ ) the lamination $E_{\mathrm{r}}(\lambda)$ spirals in the positive way around $c_{i}$. Thus, if you take a point $\left(\eta, \epsilon_{1}, \ldots, \epsilon_{n}\right) \in U$ then the corresponding $\bar{\epsilon}_{i}$ is equal to $\varepsilon$.

There is a corresponding definition of left earthquake, and we call $E_{1}: \mathcal{M} \mathcal{L}_{g, n} \times$ $\hat{\mathcal{T}}_{g, n} \rightarrow \hat{\mathcal{T}}_{g, n}$. It follows directly from the definition that, for all $\lambda \in \mathcal{M} \mathcal{L}_{g, n}, E_{1}(\lambda)=E_{\mathrm{r}}(\lambda)^{-1}$. As already mentioned above, Definition 9.4 has some desirable properties that cannot easily be achieved by more simple-looking definitions.

Proposition 9.6. The map $E_{\mathrm{r}}: \mathcal{M} \mathcal{L}_{g, n} \times \hat{\mathcal{T}}_{g, n} \rightarrow \hat{\mathcal{T}}_{g, n}$ is continuous.

Proof. We have seen above that the variation of the length of a boundary component under $E_{\mathrm{r}}(\lambda)$ is proportional to the mass of $\lambda$. The proof is therefore a direct consequence of the definition of the topology on $\hat{\mathcal{T}}_{g, n}$.

Proposition 9.7. Let $\lambda \in \mathcal{M} \mathcal{L}_{g, n}$, and let $t, t^{\prime} \in \mathbb{R}_{>0}$. Then $E_{\mathrm{r}}\left(\left(t+t^{\prime}\right) \lambda\right)=E_{\mathrm{r}}\left(t^{\prime} \lambda\right) \circ$ $E_{\mathrm{r}}(t \lambda)$.

Proof. Let $\left(h, \epsilon_{1}, \cdots, \epsilon_{n}\right) \in \hat{\mathcal{T}}_{g, n}$, and let $\left(h^{\prime}, \epsilon_{1}^{\prime}, \cdots, \epsilon_{n}^{\prime}\right)=E_{\mathrm{r}}(t \lambda)\left(h, \epsilon_{1}, \cdots, \epsilon_{n}\right)$. Let $\bar{\lambda}$ be the image of $\lambda$ under the reflection relative to all boundary components of $S$ for which $\epsilon_{i}=$ -1 . Suppose first that $\forall i \in\{1, \cdots, n\}, \epsilon_{i}^{\prime}=\epsilon_{i}$. This implies that, after the right earthquake $E_{\mathrm{r}}(t \lambda)$ (considered as an earthquake acting on $\left.\hat{\mathcal{T}}_{g, n}\right) \bar{\lambda}$ spirals in the same direction on to each of the boundary components of $S$. In other terms, $\bar{\lambda}$ remains the same measured lamination after the earthquake $E_{\mathrm{r}}(\lambda)$. The fact that

$$
E_{\mathrm{r}}\left(t^{\prime} \lambda\right) \circ E_{\mathrm{r}}(t \lambda)\left(h, \epsilon_{1}, \cdots, \epsilon_{n}\right)=E_{\mathrm{r}}\left(\left(t+t^{\prime}\right) \lambda\right)\left(h, \epsilon_{1}, \cdots, \epsilon_{n}\right)
$$

then follows directly from the definition of an earthquake (through the right-quake cocycle as seen in Section 3).

Suppose now that some of the $\bar{\epsilon}_{i}$ are different from the corresponding $\epsilon_{i}$, and let $\bar{\lambda}^{\prime}$ be the image of $\lambda$ under the reflection relative to all boundary components of $S$ for which $\bar{\epsilon}_{i}=-1$. The definition of the image of an element of $\hat{\mathcal{T}}_{g, n}$ implies that $\bar{\lambda}^{\prime}$ is the image of $\bar{\lambda}$ under the earthquake $E_{\mathrm{r}}(t \lambda)$-the boundary components of $S$ for which $\bar{\lambda}$ and $\bar{\lambda}^{\prime}$ circle in opposite directions are precisely those for which $\bar{\epsilon}_{i} \neq \epsilon_{i}$. Thus the result follows again from standard facts on earthquakes. 
Proof of Theorem 1.4. The results of Section 8 show that there are $2^{k}$ left earthquakes sending a given hyperbolic metric (considered as an element of $\mathcal{T}_{g, n}$ ) to another one, where $k$ is the number of punctures corresponding to geodesic boundary components in both metrics. The corresponding measured laminations are the bending lamination of the future boundary of a convex retract $U$ of $M$ which has as boundary curve an extremal curve (see Proposition 8.1). It was also noted (in Proposition 8.2) that each boundary curve is the upper boundary curve when the (signed) mass $m$ of the measured lamination at the corresponding boundary component of $F$ is bigger than $a$, the length of that boundary component in the induced metric on the bent surface in $M$. However, we have seen in Section 3 that $m>a$ if and only if the lamination $\lambda$ spirals in the opposite direction on that boundary component after the left earthquake is performed. So each upper extremal curve corresponds to a boundary component for which the spiraling orientation is reversed by the earthquake along $\lambda$, while each lower extremal curve corresponds to a boundary component for which the spiraling orientation remains the same. This proves Theorem 1.4.

\section{Multi-black Holes}

By now, we have studied the action of $h$ on the boundary of $\mathrm{AdS}_{3}$. Let us now consider the action of $h$ on $\mathrm{AdS}_{3}$. A first easy remark is that such an action is neither proper nor causal. For instance, the light-like plane $P$ which is tangent to the boundary at infinity of $\mathrm{AdS}_{3}$ at $p_{++}(\gamma)$ (considered in the projective model of $\mathrm{AdS}_{3}$ ) is preserved by $h(\gamma)$, and the orbits of $h(\gamma)$ on $P$ are contained in light-like rays.

In $[3,4]$, it has been shown that there exists a maximal domain, say $\Omega=\Omega\left(h_{1}, h_{\mathrm{r}}\right)$ of $\mathrm{AdS}_{3}$ such that the action of $h$ on $\Omega$ is free and properly discontinuous and the quotient $\Omega / h\left(\pi_{1}(\Sigma)\right)$ is a strongly causal Lorentzian manifold homeomorphic to $\Sigma \times \mathbb{R}$. We will refer to this quotient as a MBH spacetime $M=M(h)=\Omega / h\left(\pi_{1}(\Sigma)\right)$.

Let $\mathcal{K}$ be the convex hull of the limit set $\Lambda$ in $\mathrm{AdS}_{3}$. Recall that given an oriented space-like plane $P$ in $\mathrm{AdS}_{3}$, all time-like geodesic planes orthogonal to $P$ intersect at distance exactly $\pi / 2$ from $P$. The intersection point on the positive side of $P$ is called the point dual to $P$. Using this notion, $\Omega$ can be defined as the set of points whose dual planes are disjoint from $\mathcal{K}$ (see $[3,4]$ for details). 
Let us collect some properties of $\Omega$ :

(1) It is convex and strongly causal.

(2) The intersection with the boundary at infinity of $\mathrm{AdS}_{3}$ of the closure of $\Omega$ is the asymptotic region $\mathcal{G}$ of $h$, as described in the previous subsection.

(3) It contains the convex core of $\mathcal{K}$.

Notice that $\Omega$ is not globally hyperbolic. Any globally hyperbolic spacetime with holonomy equal to $h$ isometrically embeds into $\Omega$. Thus, $\Omega$ can also be described as the union of all $h$-invariant globally hyperbolic domains. Such domains, in turn, are in oneto-one correspondence with $h$-invariant nowhere time-like closed curves in $\partial \mathrm{AdS}_{3}$. Let us note that $\mathcal{K}$ is contained in the convex hull of every such closed curve in $\partial \mathrm{AdS}_{3}$.

The "black hole" of $\Omega$ is, by definition, the set of points that cannot be connected to $\mathcal{G}$ along any future-directed causal path (i.e., the domain in $\Omega$ that is causally disconnected from the "infinity" $\mathcal{G}$ in the future). Barbot $[3,4]$ pointed out that this set is globally hyperbolic and corresponds to the extremal curve in the boundary obtained by choosing the arc $\wedge$ in each $\mathcal{G}(\gamma)$.

There is also a "white hole," that is the set of points that cannot be connected to $\mathcal{G}$ along any past-directed causal curve. It is the globally hyperbolic domain whose boundary at infinity is obtained by choosing the extremal arc $\vee$ in each $\mathcal{G}(\gamma)$.

The intersection of the black hole and the white hole is the set of points disconnected from $\mathcal{G}$ both in the future and in the past. It can be regarded as the set of points contained in all $h$-invariant globally hyperbolic domains (in particular it contains $\mathcal{K}$ ).

Notice that for each $\gamma \in \pi_{1}(\Sigma)$ corresponding to a non-degenerate asymptotic region, the geodesic, say $c_{\gamma}$, joining $p_{--}(\gamma)$ to $p_{++}(\gamma)$ in $\operatorname{AdS}_{3}$ is contained in the boundary of $\mathcal{K}$. This geodesic is contained in the light-like planes dual to $p_{+-}(\gamma)$ and $p_{-+}(\gamma)$. Consider then the light-like triangles with base $c_{\gamma}$ and vertex, respectively, in $p_{-+}(\gamma)$ and $p_{+-}(\gamma)$. The union of these triangles disconnects $\Omega$ in two regions, the one that faces $\mathcal{G}(\gamma)$ is called the asymptotic region of $\gamma$ in $\Omega$.

The union of such triangles disconnects $\Omega$ in an "internal" piece, that is $\hat{\Omega}$, and a certain set of regions that faces the non-degenerate AR's. We call such regions the asymptotic regions of $\Omega$. The asymptotic regions of the $\mathrm{MBH}$ spacetime are defined as the corresponding quotients.

The domain $\hat{\Omega}$ turns out to be the union of the black hole and the white hole. Thus, the boundary of $\hat{M}=\hat{\Omega} / h$ is formed by $k$ annuli, each of which is the union of two light-like totally geodesic annuli along a space-like geodesics. Notice that $\hat{M}$ is a strong deformation retract of $M$. 
The set of geodesics $\left\{c_{\gamma}\right\}$ is contained in the boundary of $\partial \mathcal{K}$. They disconnect the boundary in two bent surfaces, the upper and the lower boundaries of $\partial \mathcal{K}$. The intrinsic metric on them is hyperbolic, and in fact they are isometric to some straight convex sets of $\mathbb{H}^{2}$. Moreover, the bending gives rise to a measured geodesic lamination on each. Clearly, $\partial_{ \pm} \mathcal{K}$ is invariant under the action of $h$, and $\partial_{ \pm} \mathcal{K} / h$ produces an admissible structure.

\section{Some Remarks}

Ends versus cone singularities. The statements presented here, concerning earthquakes on hyperbolic surfaces with geodesic boundary, can quite naturally be compared to corresponding results on closed surfaces endowed with hyperbolic metrics with cone singularities (as in [9]). Indeed, cone singularities can in a fairly natural way be considered as analytic continuations of geodesic boundary components when the length becomes imaginary. Another way to state the relation between the two is that black holes (or more precisely, singularities inside them, i.e., bending lines on the boundary of $M$ ) are "particles" moving along space-like geodesics.

However, this analogy has limits. One of them is that the "Earthquake Theorem" of [9] keeps the angle at the cone singularities fixed, so that two metrics are related by a unique right earthquake and there is no analog of the appearance of the enhanced Teichmüller space, which is a key feature for hyperbolic surfaces with geodesic boundary.

There might very well be a statement generalizing both the main result here and the main result of [9], and describing the earthquakes between two hyperbolic metrics having both cone singularities (of angle less than $\pi$ ) and geodesic boundary components. One could even imagine a proof based on a Mess type parameterization, by a right and left hyperbolic metric, of the space of multi-black holes of a given topology containing "particles" of fixed cone angle.

Other possible proofs. There are at least two possible proofs of Thurston's Earthquake Theorem for (smooth) hyperbolic metrics on closed surfaces, in addition to the Mess argument used above. One, originating in the work of Kerckhoff [16], uses analytic properties of the lengths of closed geodesics under earthquakes. The other, due to Thurston [20], uses more geometric constructions to construct an earthquake from an orientation-preserving homeomorphism from $S^{1}$ to itself.

It appears quite likely that those arguments can be extended to provide other proofs of the "Earthquake Theorem" presented here for hyperbolic surfaces with geodesic boundary components. The proof given by Thurston, in particular, might well 
extend to the case under consideration, however not in a completely straightforward way since one would have to construct appropriate homeomorphisms of $S^{1}$ from two hyperbolic metrics with geodesic boundary and then understand the boundedness of the earthquake obtained through Thurston's theorem.

More precisely, given two hyperbolic metrics $g_{1}$ and $g_{2}$ with geodesic boundary on a compact surface with boundary $\bar{\Sigma}$, they define an equivariant self-homeomorphism of the boundary at infinity of $\Sigma$, which is a Cantor set in $S^{1}$. Extending this homeomorphism to an equivariant map from $S^{1}$ to itself can be done in many ways. In particular, there are $2^{k}$ such extensions-where $k$ is the number of boundary components of $\bar{\Sigma}$ corresponding to geodesic boundary components (rather than cusps) for both $g_{1}$ and $g_{2}$-obtained by sending each interval in the complement of the Cantor set to either of its endpoints. It is quite conceivable that those maps are the boundary values of the earthquakes considered here.

In fact, this strategy is not really different from the one we have considered in this paper. The main point of [20] to construct a left earthquake extending a homeomorphism $\varphi$ of $S_{\infty}^{1}$ is to consider the set $\mathcal{S}$ of elements $g$ in $P S L(2, \mathbb{R})$ such that $g \circ \varphi$ is an extremal homeomorphism. The convex hulls in $\mathbb{H}^{2}$ of the fixed points of $g \circ \varphi$ are the strata for the lamination that provides the earthquake.

Instead, the key point in [17] was to consider the future boundary, $\partial_{+} \mathcal{K}$, of the convex hull in $\mathrm{AdS}_{3}$ of the graph of $\varphi$. By means of the product structure of the boundary of $\mathrm{AdS}_{3}$, two maps were pointed out $M_{L}, M_{R}: \partial_{+} \mathcal{K} \rightarrow P$, where $P \cong \mathbb{H}^{2}$ is a fixed spacelike plane. Those maps are determined by the following requirements:

(1) the restriction on each face is a projective map;

(2) ideal points of each face are sent to points on the same left (resp. right) leaf.

It turns out that $M_{L}$ is a left earthquake and $M_{R}$ is a right earthquake along the bending lamination of $\partial_{+} \mathcal{K}$ and $M_{L} \circ M_{R}^{-1}$ is the earthquake extending $\varphi$.

With the AdS language, the set $\mathcal{S}$ could be identified to the set of points whose dual plane is a support plane of $\mathcal{K}$ touching $\partial_{+} \mathcal{K}$. Moreover, the intersection of the dual plane with the future boundary is sent by $M_{R}$ to the convex core of the fixed points of $g \circ \varphi$.

Let us stress that this relation between these different proofs was already known by Mess (see the discussion in Section 7 of [17]).

Other questions. Many of the questions which are still open for globally hyperbolic AdS manifolds (and/or for quasifuchsian hyperbolic manifolds) can also be considered in 
the setting of multi-black holes. For instance, Mess [17] asked whether any couple of hyperbolic metrics can be uniquely obtained as the induced metric on the boundary of the convex core; this might be true also for hyperbolic metrics with geodesic boundary in the context of multi-black holes. The corresponding questions for the measured bending laminations of the boundary of the convex core are also of interest.

\section{Acknowledgements}

We would like to thank Thierry Barbot and Francis Bonahon for some useful conversations and comments.

\section{Funding}

F. B. was partially supported by CNRS, ANR GEODYCOS. K. K. was supported by an EPSRC Advanced Fellowship. J.-M. S. was partially supported by the ANR programs RepSurf, 2006-09, ANR06-BLAN-0311, GeomEinstein, 2006-09, 06-BLAN-0154, and ETTT (NT09-512070).

\section{References}

[1] Åminneborg, S., I. Bengtsson, D. Brill, S. Holst, and P. Peldán. “Black holes and wormholes in $2+1$ dimensions." Classical Quantum Gravity 15, no. 3 (1998): 627-44.

[2] Andersson, L., T. Barbot, R. Benedetti, F. Bonsante, W. M. Goldman, F. Labourie, K. P. Scannell, and J.-M. Schlenker. "Notes on: "Lorentz spacetimes of constant curvature" Geometriae Dedicata 126 (2007): 3-45 by G. Mess." Geometriae Dedicata 126 (2007): 47-70.

[3] Barbot, T. "Causal properties of AdS-isometry groups. I. Causal actions and limit sets." Advances in Theoretical and Mathematical Physics 12, no. 1 (2008): 1-66.

[4] Barbot, T. "Causal properties of AdS-isometry groups. II. BTZ multi-black-holes." Advances in Theoretical and Mathematical Physics 12, no. 6 (2008): 1209-57.

[5] Benedetti, R., and F. Bonsante. "Canonical Wick rotations in 3-dimensional gravity." Memoirs of the American Mathematical Society 198 (2009): 164, math.DG/0508485.

[6] Bers, L. "Simultaneous uniformization." Bulletin of the American Mathematical Society 66 (1960): 94-7.

[7] Bonahon, F., and X. Liu.“Representations of the quantum Teichmüller space and invariants of surface diffeomorphisms." Geometry \& Topology 11 (2007): 889-937.

[8] Bonsante, F., K. Krasnov, and J.-M. Schlenker. "Multi black holes and earthquakes on Riemann surfaces with boundaries." (2006): preprint math.GT/0610429, v4.

[9] Bonsante, F., and J.-M. Schlenker. "AdS manifolds with particles and earthquakes on singular surfaces." Geometric and Functional Analysis 19, no. 1 (2009): 41-82.

[10] Brill, D. "Black Holes and Wormholes in 2+ 1 Dimensions." In Mathematical and Quantum Aspects of Relativity and Cosmology, Pythagoreon, 1998, 143-79. Lecture Notes in Physics 537. Berlin: Springer, 2000. arXiv:gr-qc/9904083, MR MR1843036 (2002e:83069). 
552 F. Bonsante et al.

[11] Casson, A. J., and S. A. Bleiler. Automorphisms of Surfaces after Nielsen and Thurston. London Mathematical Society Student Texts 9. Cambridge: Cambridge University Press, 1988.

[12] Fathi, A., F. Laudenbach, and V. Poenaru. "Travaux de Thurston sur les surfaces." In Séminaire Orsay. Astérisque, no. 66-7, 284. Paris: Société Mathématique de France, 1979.

[13] Fock, V. V. "Dual Teichmüller spaces." ITEP-TH-5-1996.

[14] Fock, V. V., and L. O. Chekhov. "Quantum modular transformations, the pentagon relation, and geodesics." Trudy Matematicheskogo Instituta Imeni V. A. Steklova 226 (1999): 163-79.

[15] Fock, V. V., and L. O. Chekhov. “Quantum Teichmüller spaces." Teoreticheskaya i Matematicheskaya Fizika 120, no. 3 (1999): 511-28.

[16] Kerckhoff, S. P. “The Nielsen realization problem." Annals of Mathematics 117, no. 2 (1983): 235-65.

[17] Mess, G. "Lorentz spacetimes of constant curvature." Geometriae Dedicata 126 (2007): 3-45.

[18] O'Neill, B. “Semi-riemannian Geometry." In Pure and Applied Mathematics. With applications to relativity, 103. New York: Academic Press [Harcourt Brace Jovanovich, Publishers], 1983. xiii+468 pp. ISBN: 0-12-526740-1.

[19] Thurston, W. P. "Three-dimensional Geometry and Topology." Originally notes of lectures at Princeton University, 1979. Recent version available at http://www.msri.org/publications/ books/gt3m/, 1980.

[20] Thurston, W. P. "Earthquakes in Two-dimensional Hyperbolic Geometry." In Lowdimensional Topology and Kleinian Groups, Coventry/Durham, 1984, 91-112. London Mathematical Society Lecture Note Series 112. Cambridge: Cambridge University Press, 1986. 